\title{
A hierarchy of hydrodynamic models for plasmas. Zero-electron-mass limits in the drift-diffusion equations
}

by

\author{
Ansgar JÜNGEL ${ }^{\mathrm{a}, 1}$, Yue-Jun PENG ${ }^{\mathrm{b}, 2}$ \\ ${ }^{a}$ Fachbereich Mathematik, Technische Universität Berlin, Straße des 17. Juni 136, \\ D-10623 Berlin, Germany \\ b Laboratoire de Mathématiques Appliquées, CNRS UMR 6620, Université Blaise \\ Pascal, F-63177 Aubière Cedex, France
}

Manuscript received 4 February 1998, revised 23 November 1998

ABSTRACT. - A model hierarchy of hydrodynamic and quasi-hydrodynamic equations for plasmas consisting of electrons and ions is presented. The various model equations are obtained from the transient Euler-Poisson system for electrons and ions in the zero-electron-mass limit and/or in the zero-relaxation-time limit. A rigorous proof of the zero-electron-mass limit in the quasi-hydrodynamic equations is given. This model consists of two parabolic equations for the electrons and ions and the Poisson equation for the electric potential, subject to initial and mixed boundary conditions. The remaining asymptotic limits will be proved in forthcoming publications.

Furthermore, the existence of solutions to the limit problem which can be of degenerate type is proved without the assumptions needed for the zero-electron-mass limit (essentially, positivity of the particle densities).

\footnotetext{
${ }^{1}$ E-mail: jungel@ @math.tu-berlin.de.

${ }^{2}$ E-mail: peng@ucfma.univ-bpclermont.fr.
} 
Finally, the uniqueness of solutions to the limit problem is studied.

(C) 2000 L'Association Publications de l'Institut Henri Poincaré. Published by Elsevier B.V. All rights reserved

Key words: Plasmas, hydrodynamic equations, quasilinear parabolic equations, asymptotic limits, existence and uniqueness of solutions

AMS classification: $35 \mathrm{~K} 65,35 \mathrm{~B} 40,78 \mathrm{~A} 35$

RÉSUMÉ. - Une hiérarchie d'équations hydrodynamiques et quasihydrodynamiques pour les plasmas constitués d'électrons et ions est présentée. Les équations des modèles résultent du système Euler-Poisson non stationnaire pour les électrons et ions par une limite de masse d'électron ("zero-electron-mass-limit") et/ou par une limite de relaxation ("zero-relaxation-time-limit"). Une démonstration rigoureuse de la limite de masse d'électron dans les équations quasi-hydrodynamiques est donnée. Ce modèle consiste en deux équations paraboliques pour les densités des électrons et ions et l'équation de Poisson pour le potentiel électrique, completées par des conditions aux limites mêlées. Les autres limites asymptotiques seront démontrées dans des publications à venir.

En outre, on montre l'existence de solutions du problème limite qui peut être du type "dégénéré", sans les hypothèses utilisées pour la limite de masse d'électron (essentiellement, positivité des densités des particules). Finalement, l'unicité de solutions du problème limite est étudiée.

(C) 2000 L'Association Publications de l'Institut Henri Poincaré. Published by Elsevier B.V. All rights reserved

\section{INTRODUCTION}

The mathematical study of Euler-Poisson systems for plasmas has attracted a lot of attention in the mathematical literature since several years (see, e.g., [4,5,20-25]). In order to perform numerical simulations of the hyperbolic equations, a lot of computing power and special algorithms are needed $[9,12]$. In some situations, however, the model equations can be approximated by simpler equations, like drift-diffusion models, in the sense that a small parameter appearing in the hyperbolic equations is set equal to zero. Considering a plasma composed of electrons and ions, the small parameters are, e.g., the electron mass ("zero-electron-mass limit") or the relaxation time ("zero-relaxation-time 
limit"). Therefore, letting the small parameters tend to zero we obtain a hierarchy of hydrodynamic and quasi-hydrodynamic plasma models.

We want to present this model hierarchy, make precise the connections between the corresponding systems, and prove rigorously the asymptotic limits as the small parameters tend to zero. In this paper we are concerned with the zero-electron-mass limit in the drift-diffusion equations and with the existence and uniqueness of solutions of the limit equations. The zero-relaxation-time limits and the zero-electron-mass limit in the hydrodynamic equations will be proved in forthcoming publications [10, 17].

We consider an unmagnetized plasma consisting of electrons with mass $m_{e}$ and charge $q_{e}=-1$ and of a single species of ions with mass $m_{i}$ and charge $q_{i}=+1$. Denoting by $n_{e}=n_{e}(x, t), u_{e}=u_{e}(x, t)$ (respectively, $n_{i}, u_{i}$ ) the scaled density and mean velocity of the electrons (respectively, ions) and by $\phi=\phi(x, t)$ the scaled electric potential, these variables satisfy the following scaled Euler-Poisson system (HD-EI):

$$
\begin{aligned}
& \partial_{t} n_{\alpha}+\operatorname{div}\left(n_{\alpha} u_{\alpha}\right)=0, \\
& \delta_{\alpha} \partial_{t}\left(n_{\alpha} u_{\alpha}\right)+\delta_{\alpha} \operatorname{div}\left(n_{\alpha} u_{\alpha} \otimes u_{\alpha}\right)+\nabla p_{\alpha}\left(n_{\alpha}\right) \\
& \quad=-q_{\alpha} n_{\alpha} \nabla \phi-\delta_{\alpha} \frac{n_{\alpha} u_{\alpha}}{\tau_{\alpha}}, \\
& -\lambda^{2} \Delta \phi=n_{i}-n_{e},
\end{aligned}
$$

where $\alpha=e, i$ and $(x, t) \in \mathbb{R}^{d} \times(0, \infty)$. This system is complemented by initial conditions for $n_{\alpha}$ and $u_{\alpha}$ and by a boundary condition for $\phi$. Here, $u_{\alpha} \otimes u_{\alpha}$ denotes the tensor product with components $u_{\alpha, j} u_{\alpha, k}$ for $j, k=1, \ldots, d, \lambda>0$ is the scaled Debye length, and $\tau_{e}>0$ and $\tau_{i}>0$ are the scaled relaxation time constants for electrons and ions, respectively.

The pressure functions are usually of the form

$$
p_{\alpha}\left(n_{\alpha}\right)=a_{\alpha}^{2} n_{\alpha}^{\gamma_{\alpha}}, \quad n_{\alpha} \geqslant 0
$$

where $\gamma_{\alpha} \geqslant 1$ and $a_{\alpha}>0$. The fluid is called isothermal if $\gamma_{\alpha}=1$ ( $\alpha=e$ or $\alpha=i$ ) and adiabatic if $\gamma_{\alpha}>1$. In this paper we only assume that $p_{\alpha}$ is a strictly increasing function, which includes both cases. The system (1.1)-(1.3) is studied in $[4,20,22,24]$ when $d=1$.

The dimensionless parameters $\delta_{\alpha}$ are given by

$$
\delta_{\alpha}=\frac{m_{\alpha} v_{0}^{2}}{k_{B} T_{0}}, \quad \alpha=e, i,
$$


where $k_{B}$ is the Boltzmann constant and $v_{0}$ and $T_{0}$ are typical velocity and temperature values for the plasma. We refer to Appendix A for details of the scaling and the physical assumptions.

Usually, the ions are heavy compared to the electrons, i.e., $m_{i} \gg m_{e}$. Therefore, if $v_{0}^{2}$ is equal to $k_{B} T_{0} / m_{i}$, we get

$$
\delta_{i}=1, \quad \delta_{e} \ll 1 .
$$

Letting (formally) $\delta_{e} \rightarrow 0$ in Eq. (1.2), we obtain

$$
0=\nabla p_{e}\left(n_{e}\right)-n_{e} \nabla \phi=n_{e} \nabla\left(h_{e}\left(n_{e}\right)-\phi\right),
$$

where $h_{\alpha}(\alpha=e, i)$ are the enthalpy functions defined by

$$
h_{\alpha}^{\prime}(s)=p_{\alpha}^{\prime}(s) / s, \quad s>0, \quad h_{\alpha}(1)=0 .
$$

Hence, if $n_{e}>0$, we conclude that $h_{e}\left(n_{e}\right)=\phi$ or, introducing the function $f_{e}=h_{e}^{-1}, n_{e}=f_{e}(\phi)$. The integration constant can be set equal to zero by choosing a reference point for the applied potential. Therefore, the system (HD-EI) reduces in the limit $\delta_{e} \rightarrow 0$ to the model (HD-I):

$$
\begin{aligned}
\partial_{t} n_{\alpha}+\operatorname{div}\left(n_{\alpha} u_{\alpha}\right) & =0, \quad \alpha=e, i, \\
\delta_{i} \partial_{t}\left(n_{i} u_{i}\right)+\delta_{i} \operatorname{div}\left(n_{i} u_{i} \otimes u_{i}\right)+\nabla p_{i}\left(n_{i}\right) & =-n_{i} \nabla \phi-\delta_{i} \frac{n_{i} u_{i}}{\tau_{i}}, \\
-\lambda^{2} \Delta \phi & =n_{i}-f_{e}(\phi) .
\end{aligned}
$$

This zero-electron-mass limit will be proved rigorously in [10]. The existence of global weak entropy solutions to (1.5)-(1.7) is shown in [5, 23] when $d=1$.

Another set of equations is obtained in the zero-relaxation-time limit of the model (HD-EI). Indeed, introduce a scaling of time $s=\tau t$ and define

$$
\begin{aligned}
& N_{\alpha}(x, s)=n_{\alpha}(x, s / \tau), \quad U_{\alpha}(x, s)=(1 / \tau) u_{\alpha}(x, s / \tau), \\
& \Phi(x, s)=\phi(x, s / \tau),
\end{aligned}
$$

where $\tau=\tau_{e}=\tau_{i}$ (for simplicity). Then Eq. (1.2) becomes, for $\alpha=e, i$,

$$
\begin{aligned}
& \tau^{2} \delta_{\alpha} \partial_{s}\left(N_{\alpha} U_{\alpha}\right)+\tau^{2} \delta_{\alpha} \operatorname{div}\left(N_{\alpha} U_{\alpha} \otimes U_{\alpha}\right)+\nabla p_{\alpha}\left(N_{\alpha}\right) \\
& \quad=-q_{\alpha} N_{\alpha} \nabla \Phi-\delta_{\alpha} N_{\alpha} U_{\alpha} .
\end{aligned}
$$

Letting (formally) $\tau \rightarrow 0$ and setting again $t=s, n_{\alpha}=N_{\alpha}, u_{\alpha}=U_{\alpha}$ and $\phi=\Phi$, we obtain the system (DD-EI): 


$$
\begin{aligned}
\delta_{\alpha} \partial_{t} n_{\alpha}-\operatorname{div}\left(\nabla p_{\alpha}\left(n_{\alpha}\right)+q_{\alpha} n_{\alpha} \nabla \phi\right) & =0, \quad \alpha=e, i, \\
-\lambda^{2} \Delta \phi & =n_{i}-n_{e} .
\end{aligned}
$$

Equations of this type are studied in $[13,16]$. Furthermore, using the relaxation-time scaling (1.8) for $\alpha=i$ and $\tau=\tau_{i}$ in Eq. (1.6) and letting $\tau \rightarrow 0$, we get the model (DD-I):

$$
\begin{aligned}
\delta_{i} \partial_{t} n_{i}-\operatorname{div}\left(\nabla p_{i}\left(n_{i}\right)+n_{i} \nabla \phi\right) & =0, \\
-\lambda^{2} \Delta \phi & =n_{i}-f_{e}(\phi) .
\end{aligned}
$$

The rigorous proofs of these zero-relaxation-time limits will be presented in [17].

This paper is devoted to the asymptotic limits $\delta_{e} \rightarrow 0$ and $\delta_{i} \rightarrow 0$ in the models (DD-EI) and (DD-I). The plasma is considered to be in a bounded domain $\Omega \subset \mathbb{R}^{d}(d \geqslant 1)$ whose boundary $\partial \Omega$ consists of two disjoint sets $\Gamma_{D}$ and $\Gamma_{N}$. The model (DD-EI) is complemented by mixed DirichletNeumann boundary conditions and initial conditions, i.e., we assume that the densities and the electric potential are given on the part $\Gamma_{D}$ of the boundary and that the remaining part $\Gamma_{N}=\partial \Omega \backslash \Gamma_{D}$ is insulating:

$$
\begin{gathered}
n_{e}=n_{D, e}, \quad n_{i}=n_{D, i}, \quad \phi=\phi_{D} \quad \text { on } \Gamma_{D} \times(0, T), \\
\nabla p_{e}\left(n_{e}\right) \cdot v=\nabla p_{i}\left(n_{i}\right) \cdot v=\nabla \phi \cdot v=0 \quad \text { on } \Gamma_{N} \times(0, T), \\
n_{e}(\cdot, 0)=n_{I, e}, \quad n_{i}(\cdot, 0)=n_{I, i} \quad \text { in } \Omega .
\end{gathered}
$$

The vector $v(x)$ is the exterior unit normal which is assumed to exist a.e. Performing the zero-electron-mass limit $\delta_{e} \rightarrow 0$ in Eq. (1.9) gives

$$
n_{e} \nabla\left(h_{e}\left(n_{e}\right)-\phi\right)=\text { const. }
$$

The constant vanishes if we prescribe boundary conditions being in thermal equilibrium (see Section 2). Then, $h_{e}\left(n_{e}\right)-\phi$ is constant (in time and space). The integration constant can be chosen to be zero by defining a reference point for the electric potential. Hence

$$
h_{e}\left(n_{e}\right)=\phi \quad \text { or } \quad n_{e}=f_{e}(\phi)
$$

and we get the system (DD-I) (see (1.11)-(1.12)) with boundary and initial conditions (1.13)-(1.15) for $n_{i}, \phi$. In particular, the limits $\tau_{e}=$ $\tau_{i} \rightarrow 0, \delta_{e} \rightarrow 0$ and the limits $\delta_{e} \rightarrow 0, \tau_{i} \rightarrow 0$ are commutative (see Fig. 1).

Finally, let us consider the limits $\delta_{e} \rightarrow 0$ and $\delta_{i} \rightarrow 0$ in the system (DD-EI). Under the condition that the boundary conditions are in thermal 


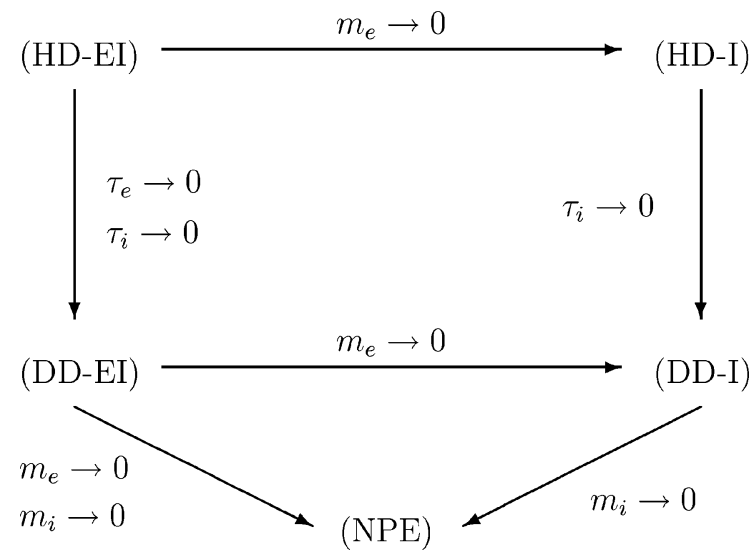

Fig. 1. A hierarchy of plasma models.

equilibrium and choosing a reference point for the electric potential, these limits in (1.9) imply

$$
h_{e}\left(n_{e}\right)-\phi=0, \quad h_{i}\left(n_{i}\right)+\phi=c,
$$

where $c \in \mathbb{R}$ is a constant. Therefore, the system (DD-EI) reduces to the following nonlinear Poisson equation (NPE):

$$
\begin{gathered}
-\lambda^{2} \Delta \phi=f_{i}(c-\phi)-f_{e}(\phi) \quad \text { in } \Omega, \\
\phi=\phi_{D} \quad \text { on } \Gamma_{D}, \quad \nabla \phi \cdot v=0 \quad \text { on } \Gamma_{N} .
\end{gathered}
$$

This equation can also be obtained from the system (DD-I) by letting (formally) $\delta_{i} \rightarrow 0$ in Eq. (1.11). We observe that the one-dimensional equation (NPE) is studied in [25]. In particular, the existence and uniqueness of solutions have been shown. A summary of the above models and limits is presented in Fig. 1.

The plan of this paper is as follows. In Section 2 we make precise the zero-electron-mass limits and present the main results. We need three main assumptions to make the limits rigorously: the strict monotonicity of the pressure functions, a positivity condition for the boundary and initial densities, and the compatibility conditions (1.16) or (1.17) on the Dirichlet boundary part. The first assumption is necessary to define the inverse function $f_{\alpha}$. From the positivity condition follows, by the maximum principle, that the electron and ion densities are strictly positive in $\Omega \times(0, T)$. Notice that for adiabatic plasmas, the parabolic 
equations (1.9) are of degenerate type and in general, solutions may exist which vanish locally.

The main results are proved in Section 3. The proofs are based on two ideas. Let us consider a solution $\left(n_{e}^{\delta}, n_{i}^{\delta}, \phi^{\delta}\right)$ to (1.9)-(1.10) subject to the boundary and initial conditions (1.13)-(1.15). First, we derive an a priori estimate independent of $\delta$ from the entropy inequality. From this estimate we get the strong convergence of $h_{e}\left(n_{e}^{\delta}\right)-\phi^{\delta}$ in $L^{2}\left(0, T ; H^{1}(\Omega)\right)$. The main difficulty then consists in showing the strong convergence of $n_{e}^{\delta}$ in $L^{2}\left(Q_{T}\right)$ in order to identify the nonlinear function. Usually, this is done by applying Aubin's lemma which requires an estimate for the time derivative of $n_{e}^{\delta}$. However, such an estimate is not available. To overcome this difficulty, we employ the monotonicity of the limiting Poisson equation (1.12) to get the strong convergence of $\phi^{\delta}$, from which we can conclude the strong convergence of $n_{e}^{\delta}$. This result is related to a compactness-by-convexity argument $[3,29]$ (see Section 3 ).

The zero-electron-mass limit $\delta_{e} \rightarrow 0$ in the system (DD-EI) provides an existence result for the model (DD-I) under the condition that positive boundary and initial conditions are prescribed. We prove in Section 4 the existence of solutions to the model (DD-I) under general assumptions on the boundary and initial conditions. The proof is based on appropriate $L^{\infty}$ estimates for $n_{i}$ and $\phi$ by using Stampacchia's truncation method. Section 5 is devoted to the proof of the uniqueness of solutions to (DDI) for isothermal pressure functions $p_{i}(s)=a_{i}^{2} s$ or for general pressure functions, but assuming positive boundary and initial densities. In the last case, the parabolic equation (1.11) is quasilinear and we employ a dual method in the uniqueness proof. Finally, in Appendix A we give the details of the scaling for the system (HD-EI).

\section{ASSUMPTIONS AND MAIN RESULTS}

This section is devoted to the study of the limits $\delta_{e} \rightarrow 0$ and $\delta_{i} \rightarrow 0$ in the drift-diffusion models (DD-EI) and (DD-I). We recall the model (DD-EI):

$$
\begin{aligned}
\delta_{e} \partial_{t} n_{e}-\operatorname{div}\left(\nabla p_{e}\left(n_{e}\right)-n_{e} \nabla \phi\right) & =0, \\
\delta_{i} \partial_{t} n_{i}-\operatorname{div}\left(\nabla p_{i}\left(n_{i}\right)+n_{i} \nabla \phi\right) & =0, \\
-\lambda^{2} \Delta \phi & =n_{i}-n_{e} \quad \text { in } \Omega \times(0, T), \\
n_{e}=n_{D, e}, \quad n_{i}=n_{D, i}, \quad \phi & =\phi_{D} \quad \text { on } \Gamma_{D} \times(0, T),
\end{aligned}
$$




$$
\begin{gathered}
\nabla p_{e}\left(n_{e}\right) \cdot v=\nabla p_{i}\left(n_{i}\right) \cdot v=\nabla \phi \cdot v=0 \quad \text { on } \Gamma_{N} \times(0, T), \\
n_{e}(\cdot, 0)=n_{I, e}, \quad n_{i}(\cdot, 0)=n_{I, i} \quad \text { in } \Omega .
\end{gathered}
$$

For convenience, we introduce the abbreviations

$$
\begin{gathered}
Q_{T}=\Omega \times(0, T), \\
V=H_{0}^{1}\left(\Omega \cup \Gamma_{N}\right)=\left\{u \in H^{1}(\Omega): u=0 \text { on } \Gamma_{D}\right\}
\end{gathered}
$$

$[28$, p. 67] and

$$
W^{m, p}(X)=W^{m, p}(0, T ; X)
$$

if $X$ is a Banach space. The norm of $W^{m, p}(\Omega)$ is denoted by $\|\cdot\|_{m, p, \Omega}$. We write $\nabla u \in L^{p}(\Omega)$ instead of $\nabla u \in L^{p}\left(\Omega ; \mathbb{R}^{d}\right)$, etc. Furthermore, we set

$$
L_{+}^{\infty}(\Omega)=\left\{u \in L^{\infty}(\Omega): u \geqslant 0 \text { a.e. in } \Omega\right\} .
$$

We say that $\left(n_{e}, n_{i}, \phi\right)$ is a weak solution to (2.1)-(2.6) if $n_{e}, n_{i} \in$ $H^{1}\left(V^{*}\right) \cap L_{+}^{\infty}\left(Q_{T}\right)$,

$$
p_{\alpha}\left(n_{\alpha}\right)-p_{\alpha}\left(n_{D, \alpha}\right) \in L^{2}(V), \quad \phi-\phi_{D} \in L^{2}(V), \quad \alpha=e, i,
$$

Eqs. (2.1)-(2.3) are satisfied in the usual variational formulation, and the initial condition is satisfied in the sense of $V^{*}$.

In $[13,16]$ the existence of global weak solutions to $(2.1)-(2.6)$ is shown under the following assumptions:

(A1) Domain: $\Omega \subset \mathbb{R}^{d}(d \geqslant 1)$ is a bounded domain with Lipschitzian boundary $\partial \Omega=\Gamma_{D} \cup \Gamma_{N}, \Gamma_{D} \cap \Gamma_{N}=\emptyset$, meas $_{d-1}\left(\Gamma_{D}\right)>0$, and $\Gamma_{N}$ is open in $\partial \Omega$.

(A2) Pressure functions: $p_{e}, \quad p_{i} \in C^{1}([0, \infty))$ are non-decreasing functions.

(A3) Initial and boundary data:

$$
\begin{aligned}
& \quad n_{D, \alpha} \in C^{0}\left([0, T] ; L_{+}^{\infty}(\Omega)\right) \cap H^{1}\left(Q_{T}\right), \quad n_{I, \alpha} \in L_{+}^{\infty}(\Omega), \\
& \text { where } \alpha=e, i ; \phi_{D} \in L^{\infty}\left(Q_{T}\right) \cap H^{1}\left(0, T ; H^{1}(\Omega)\right) .
\end{aligned}
$$

THEOREM 2.1. - Let the assumptions (A1)-(A3) hold and let $T>0$. Then there exists a weak solution $\left(n_{e}, n_{i}, \phi\right)$ to $(2.1)-(2.6)$.

If, additionally, $\partial \Omega=\Gamma_{D} \in C^{2+\varepsilon}(\varepsilon>0), \phi_{D} \in L^{\infty}\left(W^{2, q}\right)$ for some $q>d, p_{e}$ and $p_{i}$ are strictly increasing, and either

(i) $p_{\alpha}^{\prime}(s) \geqslant p_{0}>0$ for all $s \geqslant 0, \alpha=e, i$, or 
(ii) $n_{D, \alpha} \geqslant \underline{n}>0$ on $\Gamma_{D} \times(0, T), n_{I, \alpha} \geqslant \underline{n}>0$ in $\Omega, \alpha=e, i$, holds, then there exists a unique solution to (2.1)-(2.6) in the class of weak solutions satisfying $\phi \in L^{\infty}\left(W^{2, q}\right)$. Furthermore, it holds $n_{\alpha} \geqslant \underline{n}$ a.e. in $Q_{T}, \alpha=e, i$.

For the main results of this section, we need the following additional assumptions:

(A4) Positivity assumption: $n_{D, \alpha} \geqslant \underline{n}>0$ on $\Gamma_{D} \times(0, T), n_{I, \alpha} \geqslant \underline{n}>$ 0 in $\Omega, \alpha=e, i$.

$(\mathrm{A} 5)_{\alpha}$ Strictly increasing pressure: $p_{\alpha}^{\prime}(s) \geqslant \beta>0$ for all $s \in[\underline{n}, \infty)$.

(A6) $)_{e}$ Compatibility condition: $n_{D, e}=h_{e}^{-1}\left(\phi_{D}\right)$.

(A6) $)_{i}$ Compatibility condition: $n_{D, i}=h_{i}^{-1}\left(c-\phi_{D}\right)$ and

$$
c=h_{e}\left(n_{D, e}\right)+h_{i}\left(n_{D, i}\right)=\text { const. }
$$

The assumption (A4) is necessary to show that the electron and ion densities are strictly positive. From (A5) $)_{\alpha}$ follows that $h_{\alpha}^{\prime}$ is strictly positive on $[\underline{n}, \infty)$ and hence, $h_{\alpha}$ is a $C^{1}$-diffeomorphism on $[\underline{n}, \infty)$. The condition $(\mathrm{A} 6)_{\alpha}$ means that the boundary density is in thermal equilibrium. Indeed, in thermal equilibrium it holds

$$
0=\nabla p_{\alpha}\left(n_{\alpha}\right)+q_{\alpha} n_{\alpha} \phi=n_{\alpha} \nabla\left(h_{\alpha}\left(n_{\alpha}\right)+q_{\alpha} \phi\right)
$$

recalling that $h_{\alpha}^{\prime}(s)=p_{\alpha}^{\prime}(s) / s$ (see (1.4)) and $n_{\alpha} \geqslant \underline{n}$ (see Theorem 2.1). Therefore, $h_{\alpha}\left(n_{\alpha}\right)+q_{\alpha} \phi=$ const. in $Q_{T}$. By choosing a reference point for $\phi$, we get

$$
h_{e}\left(n_{e}\right)-\phi=0 \quad \text { and } \quad h_{i}\left(n_{i}\right)+\phi=c \in \mathbb{R} \text {, }
$$

or $n_{e}=h_{e}^{-1}(\phi)$ and $n_{i}=h_{i}^{-1}(c-\phi)$. Adding Eq. (2.7), we see that it must hold $h_{e}\left(n_{e}\right)+h_{i}\left(n_{i}\right)=c=$ const.

Now we can state the main theorems.

THEOREM $2.2((\mathrm{DD}-\mathrm{EI}) \rightarrow(\mathrm{DD}-\mathrm{I}))$. - Let the hypotheses (A1)-(A6) for $\alpha=e$ hold. Let $\left(n_{e}^{\delta}, n_{i}^{\delta}, \phi^{\delta}\right)$ be a solution to (2.1)-(2.6). Then there exists a subsequence $\left(n_{e}^{\delta^{\prime}}, n_{i}^{\delta^{\prime}}, \phi^{\delta^{\prime}}\right)$ of $\left(n_{e}^{\delta}, n_{i}^{\delta}, \phi^{\delta}\right)$ such that, as $\delta^{\prime} \rightarrow 0$, 


$$
\begin{aligned}
n_{e}^{\delta^{\prime}} \rightarrow f_{e}(\phi) & \text { in } L^{2}\left(Q_{T}\right), \\
& n_{i}^{\delta^{\prime}} \rightarrow n_{i} \quad \text { in } L^{2}\left(Q_{T}\right), \\
& n_{i}^{\delta^{\prime}} \rightarrow n_{i} \quad \text { weakly in } L^{2}\left(H^{1}\right) \cap H^{1}\left(V^{*}\right), \\
\phi^{\delta^{\prime}} \rightarrow \phi & \text { in } L^{2}\left(H^{1}\right),
\end{aligned}
$$

where $\left(n_{i}, \phi\right) \in L^{\infty}\left(Q_{T}\right)^{2}$ solves the initial-boundary value problem (1.11)-(1.12), (2.4)-(2.6) for $n_{i}$ and $\phi$.

In the case, where this limit problem is uniquely solvable (for instance, if $p_{i}(s)=s$; see Section 5), the above convergence results hold for the whole sequence $\left(n_{e}^{\delta}, n_{i}^{\delta}, \phi^{\delta}\right)$.

THEOREM $2.3((\mathrm{DD}-\mathrm{EI}) \rightarrow(\mathrm{NPE}))$. - Let the hypotheses (A1)-(A6) for $\alpha=e, i$ hold and let (for simplicity) $\delta=\delta_{e}=\delta_{i}$. Let $\left(n_{e}^{\delta}, n_{i}^{\delta}, \phi^{\delta}\right)$ be a solution to (2.1)-(2.6). Then, as $\delta \rightarrow 0$,

$$
\begin{aligned}
n_{e}^{\delta} \rightarrow f_{e}(\phi) & \text { in } L^{2}\left(Q_{T}\right), \\
n_{i}^{\delta} \rightarrow f_{i}(c-\phi) & \text { in } L^{2}\left(Q_{T}\right), \\
\phi^{\delta} \rightarrow \phi & \text { in } L^{2}\left(H^{1}\right),
\end{aligned}
$$

where $\phi$ is a solution of the nonlinear Poisson equation (1.18)-(1.19).

THEOREM $2.4((\mathrm{DD}-\mathrm{I}) \rightarrow(\mathrm{NPE}))$. - Let the hypotheses (A1)-(A6) for $\alpha=i$ hold and let $\left(n_{i}^{\delta}, \phi^{\delta}\right)$ be a solution to (1.11)-(1.12), (2.4)-(2.6). Then, as $\delta \rightarrow 0$,

$$
\begin{aligned}
n_{i}^{\delta} \rightarrow f_{i}(c-\phi) & \text { in } L^{2}\left(Q_{T}\right), \\
\phi^{\delta} \rightarrow \phi & \text { in } L^{2}\left(H^{1}\right),
\end{aligned}
$$

where $\phi$ is a solution of the nonlinear Poisson equation (1.18)-(1.19).

\section{PROOFS OF THE MAIN RESULTS}

We prove first Theorem 2.2. The proofs of Theorems 2.3 and 2.4 are very similar. To simplify the presentation, we set $\delta=\delta_{e}, \delta_{i}=1$ and $\lambda=1$. The following lemma provides uniform a priori estimates for the solution of (DD-EI).

LEMMA 3.1. - Assume (A1)-(A4). Then there exist positive constants $\bar{n}, \bar{\phi}$ and $K$, which are independent of $\delta$, such that 


$$
\begin{gathered}
0<\underline{n} \leqslant n_{\alpha}(x, t) \leqslant \bar{n} \quad(\alpha=e, i), \quad|\phi(x, t)| \leqslant \bar{\phi} \quad \text { in } Q_{T}, \\
\left\|n_{e}\right\|_{L^{2}\left(H^{1}\right)}+\left\|n_{i}\right\|_{L^{2}\left(H^{1}\right)}+\left\|n_{i}\right\|_{H^{1}\left(V^{*}\right)}+\|\phi\|_{L^{2}\left(H^{1}\right)} \leqslant K,
\end{gathered}
$$

where $\underline{n}>0$ is defined in (A4) (see Section 2).

Proof. - It is shown in [16] that the condition (A4) implies the positivity of $n_{e}$ and $n_{i}$ in $Q_{T}$. Therefore, $n_{e}, n_{i} \in L^{2}\left(H^{1}\right)$, and we can use $\left(n_{e}-M\right)^{+}=\max \left(0, n_{e}-M\right),\left(n_{i}-M\right)^{+}$as test functions in Eqs. (2.1) and (2.2), respectively, with

$$
M=\max \left\{\left\|n_{D, \alpha}\right\|_{0, \infty, \Gamma_{D} \times(0, T)},\left\|n_{I, \alpha}\right\|_{0, \infty, \Omega}: \alpha=e, i\right\} .
$$

Then we get, using (2.3),

$$
\begin{aligned}
& \frac{\delta}{2} \int_{\Omega}\left(n_{e}-M\right)^{+}(t)^{2}+\frac{1}{2} \int_{\Omega}\left(n_{i}-M\right)^{+}(t)^{2}+\int_{Q_{t}} p_{e}^{\prime}\left(n_{e}\right)\left|\nabla\left(n_{e}-M\right)^{+}\right|^{2} \\
& \quad+\int_{Q_{t}} p_{i}^{\prime}\left(n_{i}\right)\left|\nabla\left(n_{i}-M\right)^{+}\right|^{2} \\
& =\int_{Q_{t}}\left(n_{e}-M\right) \nabla \phi \cdot \nabla\left(n_{e}-M\right)^{+}+M \int_{Q_{t}} \nabla \phi \cdot \nabla\left(n_{e}-M\right)^{+} \\
& \quad-\int_{Q_{t}}\left(n_{i}-M\right) \nabla \phi \cdot \nabla\left(n_{i}-M\right)^{+}-M \int_{Q_{t}} \nabla \phi \cdot \nabla\left(n_{i}-M\right)^{+} \\
& =-\frac{1}{2} \int_{Q_{t}}\left(n_{e}-n_{i}\right)\left(\left(n_{e}-M\right)^{+2}-\left(n_{i}-M\right)^{+2}\right) \\
& \quad-M \int_{Q_{t}}\left(n_{e}-n_{i}\right)\left(\left(n_{e}-M\right)^{+}-\left(n_{i}-M\right)^{+}\right) \\
& \leqslant 0 .
\end{aligned}
$$

Therefore, we get the upper bound for $n_{e}$ and $n_{i}$ with $\bar{n}=M$. Using $\left(n_{\alpha}-\underline{n}\right)^{-}=\min \left(0, n_{\alpha}-\underline{n}\right)$ for $\alpha=e, i$ as test functions in (2.1)-(2.2), respectively, we get similar as above the inequality

$$
\frac{\delta}{2} \int_{\Omega}\left(n_{e}-\underline{n}\right)^{-}(t)^{2}+\frac{1}{2} \int_{\Omega}\left(n_{i}-\underline{n}\right)^{-}(t)^{2} \leqslant 0,
$$

from which we conclude the lower bound. This estimate follows from the monotonicity of the function $s \mapsto \frac{1}{2}(s-\underline{n})^{-2}+\underline{n}(s-\underline{n})^{-}=\frac{1}{2}(s-$ 
$\underline{n})^{-}(s+\underline{n}), s \geqslant 0$. The $L^{\infty}$ bounds on $n_{\alpha}$ immediately provide bounds on $\phi$, i.e., there exist $\bar{\phi}>0$ and $K>0$, independent of $\delta$, such that

$$
|\phi(x, t)| \leqslant \bar{\phi} \quad \text { for }(x, t) \in Q_{T}, \quad\|\phi\|_{L^{2}\left(H^{1}\right)} \leqslant K .
$$

To derive the remaining bounds, we use $n_{e}-n_{D, e}$ as test function in (2.1):

$$
\begin{aligned}
& \frac{\delta}{2} \int_{\Omega}\left(n_{e}-n_{D, e}\right)^{2}(t)+\int_{Q_{t}} p_{e}^{\prime}\left(n_{e}\right)\left|\nabla n_{e}\right|^{2} \\
& =\int_{Q_{t}} p_{e}^{\prime}\left(n_{e}\right) \nabla n_{e} \cdot \nabla n_{D, e}+\frac{\delta}{2} \int_{\Omega}\left(n_{I, e}-n_{D, e}(0)\right)^{2} \\
& \quad-\delta \int_{Q_{t}}\left(n_{e}-n_{D, e}\right) \partial_{t} n_{D, e}+\int_{Q_{t}} n_{e} \nabla \phi \cdot \nabla\left(n_{e}-n_{D, e}\right) .
\end{aligned}
$$

To estimate the last integral on the right-hand side, we use Eq. (2.3):

$$
\begin{aligned}
\int_{Q_{t}} & n_{e} \nabla \phi \cdot \nabla\left(n_{e}-n_{D, e}\right) \\
= & -\frac{1}{2} \int_{Q_{t}} \Delta \phi\left(n_{e}-n_{D, e}\right)^{2}+\int_{Q_{t}} n_{D, e} \nabla \phi \cdot \nabla\left(n_{e}-n_{D, e}\right) \\
= & -\frac{1}{2} \int_{Q_{t}}\left(n_{e}-n_{i}\right)\left(n_{e}-n_{D, e}\right)^{2}-\int_{Q_{t}} n_{D, e}\left(n_{e}-n_{i}\right)\left(n_{e}-n_{D, e}\right) \\
& -\int_{Q_{t}} \nabla n_{D, e} \cdot \nabla \phi\left(n_{e}-n_{D, e}\right) .
\end{aligned}
$$

Using the $L^{\infty}$ bounds on $n_{e}, n_{i}$ and the $L^{2}\left(H^{1}\right)$ bound for $\phi$ and employing Young's inequality, we obtain from (3.1) the bound

$$
\left\|n_{e}\right\|_{L^{2}\left(H^{1}\right)} \leqslant K,
$$

where $K>0$ is independent of $\delta$. We get an analogous bound for $n_{i}$ in the space $L^{2}\left(H^{1}\right)$. Finally, we have

$$
\begin{aligned}
\left\|\partial_{t} n_{i}\right\|_{L^{2}\left(V^{*}\right)} & \leqslant\left\|\nabla p_{i}\left(n_{i}\right)-n_{i} \nabla \phi\right\|_{L^{2}\left(L^{2}\right)} \\
& \leqslant\left\|p_{i}^{\prime}\left(n_{i}\right)\right\|_{L^{\infty}\left(L^{\infty}\right)}\left\|\nabla n_{i}\right\|_{L^{2}\left(L^{2}\right)}+\left\|n_{i}\right\|_{L^{\infty}\left(L^{\infty}\right)}\|\nabla \phi\|_{L^{2}\left(L^{2}\right)} \\
& \leqslant K
\end{aligned}
$$


which proves the lemma.

Now, we define the entropy (or free energy) of the system (2.1)-(2.6):

$$
\begin{aligned}
L(t) \stackrel{\text { def }}{=} & \sum_{\alpha=e}^{i} \int_{\Omega}\left(\int_{n_{D, \alpha}(t)}^{n_{\alpha}(t)}\left(h_{\alpha}(\sigma)-h_{\alpha}\left(n_{D, \alpha}(t)\right)\right) \mathrm{d} \sigma\right) \\
& +\frac{1}{2} \int_{\Omega}\left|\nabla\left(\phi(t)-\phi_{D}(t)\right)\right|^{2} .
\end{aligned}
$$

The enthalpy functions $h_{\alpha}$ are defined in (1.4). It holds $L(t) \geqslant 0$. The key estimate for the zero-electron-mass limit is contained in the following lemma.

LEMMA 3.2. - There exists a constant $c_{1}>0$, independent of $\delta$, such that for all $t \geqslant 0$

$$
L(t)+\frac{1}{\delta} \int_{Q_{t}} n_{e}\left|\nabla\left(h_{e}\left(n_{e}\right)-\phi\right)\right|^{2} \leqslant c_{1} .
$$

Proof. - It can be shown that (see, e.g., [15, p. 513])

$$
\begin{aligned}
L(t)-L(0)= & \sum_{\alpha=e}^{i} \int_{0}^{t}\left(\left\langle\partial_{t} n_{\alpha}(s), h_{\alpha}\left(n_{\alpha}(s)\right)-h_{\alpha}\left(n_{D, \alpha}(s)\right)\right\rangle\right. \\
& \left.-\int_{\Omega} \int_{n_{D, \alpha}(s)}^{n_{\alpha}(s)} h_{\alpha}^{\prime}\left(n_{D, \alpha}(s)\right) \partial_{t} n_{D, \alpha}(s) \mathrm{d} \sigma\right) \mathrm{d} s \\
& +\int_{Q_{t}} \partial_{t} \nabla\left(\phi-\phi_{D}\right) \cdot \nabla\left(\phi-\phi_{D}\right),
\end{aligned}
$$

since $\phi \in H^{1}\left(H^{1}\right)$. Using

$$
\int_{Q_{t}} \partial_{t} \nabla \phi \cdot \nabla\left(\phi-\phi_{D}\right)=\int_{0}^{t}\left\langle\partial_{t}\left(n_{i}-n_{e}\right), \phi-\phi_{D}\right\rangle,
$$

we get

$$
L(t)-L(0)=\int_{0}^{t}\left\langle\partial_{t} n_{e}, h_{e}\left(n_{e}\right)-\phi-\left(h_{e}\left(n_{D, e}\right)-\phi_{D}\right)\right\rangle
$$




$$
\begin{aligned}
& +\int_{0}^{t}\left\langle\partial_{t} n_{i}, h_{i}\left(n_{i}\right)+\phi-\left(h_{i}\left(n_{D, i}\right)+\phi_{D}\right)\right\rangle \\
& -\sum_{\alpha=e}^{i} \int_{Q_{t}} h_{\alpha}^{\prime}\left(n_{D, \alpha}\right) \partial_{t} n_{D, \alpha}\left(n_{\alpha}-n_{D, \alpha}\right) \\
& -\int_{Q_{t}} \partial_{t} \nabla \phi_{D} \cdot \nabla\left(\phi-\phi_{D}\right) .
\end{aligned}
$$

Employing Eqs. (2.1) and (2.2) and the condition (A6) $)_{e}$, the first two integrals on the right-hand side of (3.2) are equal to

$$
\begin{aligned}
& -\frac{1}{\delta} \int_{Q_{t}} n_{e}\left|\nabla\left(h_{e}\left(n_{e}\right)-\phi\right)\right|^{2}-\int_{Q_{t}} n_{i}\left|\nabla\left(h_{i}\left(n_{i}\right)+\phi\right)\right|^{2} \\
& \quad+\int_{Q_{t}} n_{i} \nabla\left(h_{i}\left(n_{i}\right)+\phi\right) \cdot \nabla\left(h_{i}\left(n_{D, i}\right)+\phi_{D}\right) \\
& \leqslant-\frac{1}{\delta} \int_{Q_{t}} n_{e}\left|\nabla\left(h_{e}\left(n_{e}\right)-\phi\right)\right|^{2}-\frac{1}{2} \int_{Q_{t}} n_{i}\left|\nabla\left(h_{i}\left(n_{i}\right)+\phi\right)\right|^{2} \\
& \quad+\frac{1}{2} \int_{Q_{t}} n_{i}\left|\nabla\left(h_{i}\left(n_{D, i}\right)+\phi_{D}\right)\right|^{2} \\
& \leqslant-\frac{1}{\delta} \int_{Q_{t}} n_{e}\left|\nabla\left(h_{e}\left(n_{e}\right)-\phi\right)\right|^{2}+c,
\end{aligned}
$$

where $c>0$ denotes a constant independent of $\delta$. Using the uniform $L^{\infty}$ bounds on $n_{e}$ and $n_{i}$, the remaining integrals on the right-hand side of (3.2) are bounded by a constant independent of $\delta$. Therefore, we obtain from (3.2)

$$
L(t)-L(0) \leqslant-\frac{1}{\delta} \int_{Q_{t}} n_{e}\left|\nabla\left(h_{e}\left(n_{e}\right)-\phi\right)\right|^{2}+c .
$$

The lemma follows.

We are now able to prove Theorem 2.2.

Proof of Theorem 2.2. - First step: Let $\left(n_{e}^{\delta}, n_{i}^{\delta}, \phi^{\delta}\right)$ be a solution to (2.1)-(2.6). Thanks to the uniform bounds on $n_{e}^{\delta}, n_{i}^{\delta}$ and $\phi^{\delta}$ of 
Lemma 3.1, the following convergence results are valid, after passing to a subsequence:

$$
\begin{aligned}
& n_{e}^{\delta^{\prime}} \rightarrow n_{e} \quad \text { weakly in } L^{2}\left(H^{1}\right), \\
& n_{i}^{\delta^{\prime}} \rightarrow n_{i} \quad \text { weakly in } L^{2}\left(H^{1}\right) \text { and in } H^{1}\left(V^{*}\right), \\
& \phi^{\delta^{\prime}} \rightarrow \phi \quad \text { weakly in } L^{2}\left(H^{1}\right) \text { and in } L^{2}\left(Q_{T}\right) \text { as } \delta^{\prime} \rightarrow 0 .
\end{aligned}
$$

The boundedness of $n_{i}^{\delta^{\prime}}$ in $L^{2}\left(H^{1}\right) \cap H^{1}\left(V^{*}\right)$ implies, by Aubin's lemma [26, p. 85, Corollary 4], the compactness of the sequence $n_{i}^{\delta^{\prime}}$ in the space $L^{2}\left(Q_{T}\right)$, i.e., there exists a subsequence (not relabeled) such that

$$
n_{i}^{\delta^{\prime}} \rightarrow n_{i} \quad \text { in } L^{2}\left(Q_{T}\right)
$$

We use the uniform $L^{\infty}$ bound on $n_{i}^{\delta^{\prime}}$ to conclude that

$$
p_{i}\left(n_{i}^{\delta^{\prime}}\right) \rightarrow p_{i}\left(n_{i}\right) \quad \text { in } L^{2}\left(Q_{T}\right)
$$

The uniform lower bound on $n_{e}^{\delta}$ from Lemma 3.1 and the entropy inequality from Lemma 3.2 imply

$$
\varepsilon_{\delta} \stackrel{\text { def }}{=} h_{e}\left(n_{e}^{\delta}\right)-\phi^{\delta} \rightarrow 0 \quad \text { in } L^{2}\left(Q_{T}\right) \text { as } \delta \rightarrow 0 .
$$

Here we have used the compatibility condition $(\mathrm{A} 6)_{e}$. The main difficulty is to show that $n_{e}=f_{e}(\phi)$.

Second step: We claim that

$$
n_{e}^{\delta}-f_{e}\left(\phi^{\delta}\right) \rightarrow 0 \quad \text { in } L^{2}\left(Q_{T}\right) \text { as } \delta \rightarrow 0 .
$$

Recall that $f_{e}$ is the inverse function of $h_{e}$. Indeed, there exists $\xi_{\delta}$ between $\phi^{\delta}$ and $\phi^{\delta}+\varepsilon_{\delta}$ (for fixed $(x, t) \in Q_{T}$ ) such that

$$
n_{e}^{\delta}-f_{e}\left(\phi^{\delta}\right)=f_{e}\left(\phi^{\delta}+\varepsilon_{\delta}\right)-f_{e}\left(\phi^{\delta}\right)=f_{e}^{\prime}\left(\xi_{\delta}\right) \varepsilon_{\delta} .
$$

Since $h_{e}$ is a $C^{1}$-diffeomorphism on $[\underline{n}, \infty)$ and the sequences $\left(\phi^{\delta}+\right.$ $\left.\varepsilon_{\delta}\right)_{\delta}=\left(h_{e}\left(n_{e}^{\delta}\right)\right)_{\delta}$ and $\left(\phi^{\delta}\right)_{\delta}$ are bounded in $L^{\infty}\left(Q_{T}\right)$, there is a constant $c>0$ independent of $\delta>0$ (and independent of $(x, t))$ such that

$$
\left|\left(h_{e}^{-1}\right)^{\prime}\left(\xi_{\delta}\right)\right| \leqslant c .
$$

Hence

$$
\left\|n_{e}^{\delta}-f_{e}\left(\phi^{\delta}\right)\right\|_{0,2, Q_{T}} \leqslant c\left\|\varepsilon_{\delta}\right\|_{0,2, Q_{T}} \rightarrow 0 \quad \text { as } \delta \rightarrow 0
$$


This proves (3.5).

In order to identify $n_{e}$ and $f_{e}(\phi)$ we need the strong convergence in $L^{2}\left(Q_{T}\right)$ of one of the sequences $n_{e}^{\delta^{\prime}}$ or $\phi^{\delta^{\prime}}$. However, since we do not have an appropriate bound for the time derivative $\partial n_{e}^{\delta^{\prime}} / \partial t$ in some space, we do not get the strong convergence of $n_{e}^{\delta^{\prime}}$, like for $n_{i}^{\delta^{\prime}}$, by application of Aubin's lemma. We prove instead the strong convergence of $\phi^{\delta^{\prime}}$ by using the Poisson equation (2.3).

Third step: We use $\phi^{\delta^{\prime}}-\phi$ as test function for the Poisson equation (2.3) to obtain

$$
\begin{aligned}
\int_{Q_{T}} & \nabla \phi^{\delta^{\prime}} \cdot \nabla\left(\phi^{\delta^{\prime}}-\phi\right) \\
= & \int_{Q_{T}}\left(n_{i}^{\delta^{\prime}}-f_{e}(\phi)\right)\left(\phi^{\delta^{\prime}}-\phi\right)-\int_{Q_{T}}\left(n_{e}^{\delta^{\prime}}-f_{e}\left(\phi^{\delta^{\prime}}\right)\right)\left(\phi^{\delta^{\prime}}-\phi\right) \\
& -\int_{Q_{T}}\left(f_{e}\left(\phi^{\delta^{\prime}}\right)-f_{e}(\phi)\right)\left(\phi^{\delta^{\prime}}-\phi\right) \\
\leqslant & \int_{Q_{T}}\left(n_{i}^{\delta^{\prime}}-f_{e}(\phi)\right)\left(\phi^{\delta^{\prime}}-\phi\right)-\int_{Q_{T}}\left(n_{e}^{\delta^{\prime}}-f_{e}\left(\phi^{\delta^{\prime}}\right)\right)\left(\phi^{\delta^{\prime}}-\phi\right),
\end{aligned}
$$

where we have employed the monotonicity of $f_{e}$. Hence

$$
\begin{aligned}
\int_{Q_{T}}\left|\nabla \phi^{\delta^{\prime}}\right|^{2} \leqslant & \int \nabla \phi^{\delta^{\prime}} \cdot \nabla \phi+\int_{Q_{T}}\left(n_{i}^{\delta^{\prime}}-f_{e}(\phi)\right)\left(\phi^{\delta^{\prime}}-\phi\right) \\
& -\int_{Q_{T}}\left(n_{e}^{\delta^{\prime}}-f_{e}\left(\phi^{\delta^{\prime}}\right)\right)\left(\phi^{\delta^{\prime}}-\phi\right) .
\end{aligned}
$$

Observing that the last two terms tend to zero as $\delta^{\prime} \rightarrow 0$, thanks to (3.3), (3.5) and the weak convergence of $\phi^{\delta^{\prime}}$ to $\phi$ in $L^{2}\left(Q_{T}\right)$, we get

$$
\limsup _{\delta^{\prime} \rightarrow 0} \int_{Q_{T}}\left|\nabla \phi^{\delta^{\prime}}\right|^{2} \leqslant \int_{Q_{T}}|\nabla \phi|^{2} .
$$

Since the weak convergence of $\nabla \phi^{\delta^{\prime}}$ gives

$$
\int_{Q_{T}}|\nabla \phi|^{2} \leqslant \liminf _{\delta^{\prime} \rightarrow 0} \int_{Q_{T}}\left|\nabla \phi^{\delta^{\prime}}\right|^{2},
$$


we obtain, together with (3.6),

$$
\lim _{\delta^{\prime} \rightarrow 0} \int_{Q_{T}}\left|\nabla \phi^{\delta^{\prime}}\right|^{2}=\int_{Q_{T}}|\nabla \phi|^{2}
$$

The convergence of the $L^{2}$ norms and the weak convergence imply the strong convergence of the sequence $\nabla \phi^{\delta}$ :

$$
\nabla \phi^{\delta^{\prime}} \rightarrow \nabla \phi \quad \text { in } L^{2}\left(Q_{T}\right)
$$

Note that this result is related to a compactness-by-convexity argument; indeed, the weak convergence of $\nabla \phi^{\delta^{\prime}}$ and the inequality (3.6) imply the strong convergence of $\nabla \phi^{\delta^{\prime}}$ in $L^{1}\left(Q_{T}\right)$, since the function $j(w)=|w|^{2}$, $w \in \mathbb{R}^{d}$, is strictly convex (see [29, Theorem 3] or [3, Theorem 1]).

By Poincaré's inequality, we get $\phi^{\delta^{\prime}} \rightarrow \phi$ in $L^{2}\left(Q_{T}\right)$. Therefore, in view of the $L^{\infty}$ bounds for $\phi^{\delta^{\prime}}$,

$$
f_{e}\left(\phi^{\delta^{\prime}}\right) \rightarrow f_{e}(\phi) \text { in } L^{2}\left(Q_{T}\right)
$$

Thus

$$
\begin{aligned}
& \int_{Q_{T}}\left|n_{e}^{\delta^{\prime}}-f_{e}(\phi)\right|^{2} \\
& \quad \leqslant 2 \int_{Q_{T}}\left(\left|n_{e}^{\delta^{\prime}}-f_{e}\left(\phi^{\delta^{\prime}}\right)\right|^{2}+\left|f_{e}\left(\phi^{\delta^{\prime}}\right)-f_{e}(\phi)\right|^{2}\right) \rightarrow 0
\end{aligned}
$$

as $\delta^{\prime} \rightarrow 0$. We conclude that $n_{e}=f_{e}(\phi)$.

Fourth step: The above convergence results are sufficient to perform the limit $\delta^{\prime} \rightarrow 0$ in Eqs. (2.2) and (2.3). Uniqueness of solutions to the limiting problem implies, as usual, the convergence of the whole sequence $\left(n_{e}^{\delta}, n_{i}^{\delta}, \phi^{\delta}\right)$.

Proof of Theorem 2.3. - Let $\delta=\delta_{e}=\delta_{i}$. Since we are looking for estimates independent of $\delta_{e}$ and $\delta_{i}$ we only obtain uniform bounds for $n_{i}$ in $L^{\infty}\left(Q_{T}\right)$ and $L^{2}\left(H^{1}\right)$. Furthermore, the entropy estimate in Lemma 3.2 has to be replaced by

$$
\frac{1}{\delta} \int_{Q_{t}}\left(n_{e}\left|\nabla\left(h_{e}\left(n_{e}\right)-\phi\right)\right|^{2}+n_{i}\left|\nabla\left(h_{i}\left(n_{i}\right)+\phi\right)\right|^{2}\right) \leqslant c_{1} .
$$


As in the proof of Theorem 2.2 we show that

$$
n_{i}^{\delta}-f_{i}\left(c-\phi^{\delta}\right) \rightarrow 0 \quad \text { in } L^{2}\left(Q_{T}\right) \text { as } \delta \rightarrow 0
$$

employing the assumption (A6) ${ }_{i}$. Therefore, using $\phi^{\delta}-\phi$ as test function in the Poisson equation (2.3) we obtain

$$
\begin{aligned}
\int_{Q_{T}} & \nabla \phi^{\delta} \cdot\left(\phi^{\delta}-\phi\right) \\
= & \int_{Q_{T}}\left(n_{i}^{\delta}-f_{i}\left(c-\phi^{\delta}\right)\right)\left(\phi^{\delta}-\phi\right)-\int_{Q_{T}}\left(n_{e}^{\delta}-f_{e}\left(\phi^{\delta}\right)\right)\left(\phi^{\delta}-\phi\right) \\
& +\int_{Q_{T}}\left(f_{i}\left(c-\phi^{\delta}\right)-f_{i}(c-\phi)\right)\left(\phi^{\delta}-\phi\right) \\
& -\int_{Q_{T}}\left(f_{e}\left(\phi^{\delta}\right)-f_{e}(\phi)\right)\left(\phi^{\delta}-\phi\right) \\
& +\int_{Q_{T}}\left(f_{i}(c-\phi)-f_{e}(\phi)\right)\left(\phi^{\delta}-\phi\right) .
\end{aligned}
$$

Using the monotonicity of $f_{e}$ and $f_{i}$ and the weak convergences of $n_{e}^{\delta}, n_{i}^{\delta}$ and $\phi^{\delta}$, we obtain as in the proof of Theorem 2.2 the inequality

$$
\limsup _{\delta \rightarrow 0} \int_{Q_{T}}\left|\nabla \phi^{\delta}\right|^{2} \leqslant \int_{Q_{T}}|\nabla \phi|^{2},
$$

from which we conclude that $\nabla \phi^{\delta} \rightarrow \nabla \phi$ in $L^{2}\left(Q_{T}\right)$. The remaining part is analogous to the proof of Theorem 2.2.

Proof of Theorem 2.4. - This proof is very similar to the proofs of Theorems 2.2 and 2.3. We get uniform bounds for $n_{i}$ in $L^{\infty}\left(Q_{T}\right)$ and $L^{2}\left(H^{1}\right)$. Furthermore,

$$
\frac{1}{\delta} \int_{Q_{t}} n_{i}\left|\nabla\left(h_{i}\left(n_{i}\right)+\phi\right)\right|^{2} \leqslant c_{1} .
$$

Thus $n_{i}^{\delta}-f_{i}\left(c-\phi^{\delta}\right) \rightarrow 0$ in $L^{2}\left(Q_{T}\right)$. The monotonicity of $f_{i}$ implies the inequality (3.7) and the rest is as in the proof of Theorem 2.2. 


\section{EXISTENCE OF SOLUTIONS}

In this section we prove the existence of solutions to the problem

$$
\begin{aligned}
\partial_{t} n-\operatorname{div}(\nabla p(n)+n \nabla \phi)=0, & \\
-\Delta \phi=n-f(\phi) & \text { in } Q_{T}=\Omega \times(0, T), \\
n=n_{D}, \quad \phi=\phi_{D} & \text { on } \Gamma_{D} \times(0, T), \\
\nabla p(n) \cdot v=\nabla \phi \cdot v=0 & \text { on } \Gamma_{N} \times(0, T), \\
n(0)=n_{I} & \text { in } \Omega .
\end{aligned}
$$

Here, $n$ denotes the ion density, $\phi$ the electric potential, $p(n)$ the pressure function for the ions, and $f(\phi)$ is the inverse of the enthalpy function for the electrons.

We impose the following assumptions:

(A2') $p \in C^{1}([0, \infty))$ is non-decreasing on $[0, \infty)$.

(A3') $f \in C^{0,1}(\mathbb{R})$ is increasing on $\mathbb{R}$ and $f(s) \rightarrow \infty$ as $s \rightarrow \infty$.

$\left(\mathrm{A} 4^{\prime}\right) n_{D} \in L_{+}^{\infty}\left(Q_{T}\right) \cap H^{1}\left(Q_{T}\right), \quad \phi_{D} \in L^{\infty}\left(H^{1}\right) \cap L^{\infty}\left(Q_{T}\right), n_{I} \in$ $L_{+}^{\infty}(\Omega)$.

The notion of weak solution is as in Section 2, i.e., $(n, \phi)$ is a weak solution to (4.1)-(4.5) if $n \in H^{1}\left(V^{*}\right) \cap L_{+}^{\infty}\left(Q_{T}\right)$,

$$
p(n)-p\left(n_{D}\right) \in L^{2}(V), \quad \phi-\phi_{D} \in L^{2}(V),
$$

Eqs. (4.1) and (4.2) are satisfied in the usual variational formulation, and the initial condition is satisfied in the sense of $V^{*}$.

THEOREM 4.1. - Let $T>0$. Under the assumptions (A1), (A2')$\left(\mathrm{A} 4^{\prime}\right)$ there exists a weak solution $(n, \phi)$ of (4.1)-(4.5) satisfying the bounds

$$
0 \leqslant n \leqslant M_{T}, \quad f(\phi) \leqslant M_{T}, \quad \phi \geqslant \underline{\phi}, \quad \text { in } Q_{T},
$$

where $\phi \stackrel{\text { def }}{=}-M_{T}-c(\Omega) \max (0, f(0)), c(\Omega)>0$ only depends on $\Omega$, and $M_{T}^{-}$is defined by

$$
M_{T}=\max \left(\left\|n_{D}\right\|_{0, \infty, \Gamma_{D} \times(0, T)}, f\left(\left\|\phi_{D}\right\|_{0, \infty, \Gamma_{D} \times(0, T)}\right),\left\|n_{I}\right\|_{0, \infty, \Omega}\right) .
$$

Furthermore, if $T=\infty$ in (A4') there exists a weak solution for all $t>0$ and the above bounds hold uniformly in $t$ provided that $T=\infty$ in the definition of $M_{T}$. 
Proof. - First step: An approximate problem. Let $T>0$, set $M=M_{T}$, let $K>M, s_{K}=\max (0, \min (s, K)), s_{M}=\max (0, \min (s, M))$ and

$$
p_{K}^{\varepsilon}(s)=p\left(s_{K}\right)+\varepsilon s, \quad s \in \mathbb{R}, \varepsilon>0 .
$$

First we solve the approximate problem

$$
\begin{aligned}
\partial_{t} n-\operatorname{div}\left(\nabla p_{K}^{\varepsilon}(n)+n_{K} \nabla \phi\right) & =0, \\
-\Delta \phi & =n_{M}-f(\phi) \quad \text { in } Q_{T}
\end{aligned}
$$

subject to the initial and boundary conditions (4.3)-(4.5). To solve this uniformly parabolic initial-boundary value problem we use Schauder's fixed point theorem. For this, let $u \in L^{2}\left(Q_{T}\right)$ and let $\phi(t) \in H^{1}(\Omega)$ for a.e. $t \in(0, T)$ be the unique solution of

$$
\begin{gathered}
-\Delta \phi(t)=u(t)_{M}-f(\phi(t)) \quad \text { in } \Omega, \\
\phi(t)=\phi_{D}(t) \quad \text { on } \Gamma_{D}, \quad \nabla \phi(t) \cdot v=0 \quad \text { on } \Gamma_{N} .
\end{gathered}
$$

The existence and uniqueness of the solution follow from the monotonicity of $f$ (cf. [19, Lemma 3.2.1, p. 36]). Then $\phi$ is (Bochner-)measurable in $(0, T)$ and $\phi \in L^{2}\left(H^{1}\right)$. The maximum principle implies $\phi \in L^{\infty}\left(Q_{T}\right)$. Now we solve the linear problem

$$
\begin{gathered}
\partial_{t} n-\operatorname{div}\left(\left(p_{K}^{\varepsilon}\right)^{\prime}(u) \nabla n\right)=\operatorname{div}\left(u_{K} \nabla \phi\right) \quad \text { in } Q_{T}, \\
n=n_{D} \quad \text { on } \Gamma_{D} \times(0, T), \quad \nabla n \cdot v=0 \quad \text { on } \Gamma_{N} \times(0, T) .
\end{gathered}
$$

Since $\operatorname{div}\left(u_{K} \nabla \phi\right) \in L^{2}\left(V^{*}\right)$ there exists a unique solution $n \in H^{1}\left(V^{*}\right) \cap$ $L^{2}\left(H^{1}\right)$ of this problem [30, Theorem 23.A, p. 424]. Therefore, the fixed point operator $S: L^{2}\left(Q_{T}\right) \rightarrow L^{2}\left(Q_{T}\right), u \mapsto n$, is well defined. Taking $\phi-\phi_{D}$ as test function in (4.10) and using Young's inequality and the monotonicity of $f$, we get

$$
\frac{1}{2} \int_{Q_{T}}\left|\nabla\left(\phi-\phi_{D}\right)\right|^{2} \leqslant \frac{1}{2} \int_{Q_{T}}\left|\nabla \phi_{D}\right|^{2}+\int_{Q_{T}}\left(u_{M}-f\left(\phi_{D}\right)\right)\left(\phi-\phi_{D}\right),
$$

which implies

$$
\int_{Q_{T}}|\nabla \phi|^{2} \leqslant c\left(M, \phi_{D}\right) .
$$

Using $n-n_{D}$ as test function in (4.11) gives after standard manipulations

$$
\frac{1}{2} \int_{\Omega}\left(n-n_{D}\right)^{2}(t)+\frac{\varepsilon}{2} \int_{Q_{t}}\left|\nabla\left(n-n_{D}\right)\right|^{2}
$$




$$
\begin{aligned}
& \leqslant c(\varepsilon)\left(\left\|\partial_{t} n_{D}\right\|_{L^{2}\left(V^{*}\right)}^{2}+\left\|\nabla n_{D}\right\|_{L^{2}\left(L^{2}\right)}^{2}+K^{2}\|\nabla \phi\|_{L^{2}\left(L^{2}\right)}^{2}\right) \\
& \leqslant c(\varepsilon, K, M) .
\end{aligned}
$$

Therefore, $n=S(u)$ lies in a bounded subset of $L^{\infty}\left(L^{2}\right) \cap L^{2}\left(H^{1}\right)$ and $\partial_{t} n$ lies in a bounded subset of $L^{2}\left(V^{*}\right)$. Hence

$$
\sup \left\{\|S(u)\|_{L^{2}\left(H^{1}\right) \cap H^{1}\left(V^{*}\right)}: u \in L^{2}\left(Q_{T}\right)\right\}<\infty .
$$

In view of standard compactness results [30, p. 450], this implies that $S\left(L^{2}\left(Q_{T}\right)\right)$ is precompact in $L^{2}\left(Q_{T}\right)$. Standard arguments show the continuity of $S$ (see, e.g., [13]). The existence of a solution $\left(n^{\varepsilon}, \phi^{\varepsilon}\right)$ to (4.8)-(4.9), (4.3)-(4.5) is a consequence of Schauder's fixed point theorem.

Second step: $L^{\infty}$ estimates. This is the main step of the proof. For convenience, we omit the index $\varepsilon$ in $\left(n^{\varepsilon}, \phi^{\varepsilon}\right)$. We show first that

$$
\phi(x, t) \leqslant k \quad \text { a.e. in } Q_{T},
$$

where $k \in \mathbb{R}$ is such that $f(k)=M$. The existence of $k$ follows from $\left(\mathrm{A}^{\prime}\right)$. Use $(\phi(t)-k)^{+}=\max (0, \phi(t)-k)$ as test function in (4.9) to get, for a.e. $t \in(0, T)$,

$$
\begin{aligned}
\int_{\Omega}\left|\nabla(\phi(t)-k)^{+}\right|^{2}= & -\int_{\Omega}(f(\phi(t))-f(k))(\phi(t)-k)^{+} \\
& +\int_{\Omega}\left(n(t)_{M}-f(k)\right)(\phi(t)-k)^{+} \\
\leqslant & \int_{\Omega}(M-f(k))(\phi(t)-k)^{+} \\
= & 0 .
\end{aligned}
$$

This implies (4.12). In particular, we get

$$
f(\phi) \leqslant M \quad \text { a.e. in } Q_{T}
$$

To find a lower bound for $\phi$, let $m \geqslant\left\|\phi_{D}\right\|_{0, \infty, \Gamma_{D} \times(0, T)}$ and use $(-\phi(t)-m)^{+}$in (4.9):

$$
\begin{aligned}
\int_{\Omega}\left|\nabla(-\phi(t)-m)^{+}\right|^{2} \\
\quad=-\int_{\Omega}(f(\phi(t))-f(-m))(\phi(t)-(-m))^{-}
\end{aligned}
$$




$$
\begin{aligned}
& +\int_{\Omega}\left(n_{M}-f(-m)\right)(\phi(t)-(-m))^{-} \\
\leqslant & -\int_{\Omega}\left(n_{M}-f(-m)\right)(-\phi(t)-m)^{+} \\
\leqslant & f(-m) \int_{\Omega}(-\phi(t)-m)^{+} \\
\leqslant & \max (0, f(0))(\operatorname{meas}(-\phi(t)>m))^{1 / 2}\left\|(-\phi(t)-m)^{+}\right\|_{1,2, \Omega},
\end{aligned}
$$

employing Hölder's inequality. Let $r>2$ be such that the embedding $H^{1}(\Omega) \hookrightarrow L^{r}(\Omega)$ is continuous. It is well known that for all $\mu>m$

$$
(\operatorname{meas}(-\phi(t)>\mu))^{1 / r}(\mu-m) \leqslant c(\Omega)\left\|\nabla(-\phi(t)-m)^{+}\right\|_{0,2, \Omega}
$$

holds [27, Chapter 4]. Therefore we get from (4.14) and Poincaré's inequality, for $\mu>m$,

$$
\operatorname{meas}(-\phi(t)>\mu) \leqslant \frac{c(\Omega)^{r} \max (0, f(0))^{r}}{(\mu-m)^{r}}(\operatorname{meas}(-\phi(t)>m))^{r / 2} \text {. }
$$

Since $r / 2>1$, we can apply Stampacchia's Lemma (see [28, Chapter 2.3] or [27, Chapter 4]) to get

$$
\begin{aligned}
& -\phi(t) \leqslant\left\|\phi_{D}\right\|_{0, \infty, \Gamma_{D} \times(0, T)}+c(\Omega) \max (0, f(0)) \\
& \text { a.e. in } \Omega, t \in(0, T) .
\end{aligned}
$$

Next we claim that

$$
0 \leqslant n(x, t) \leqslant M \quad \text { for a.e. }(x, t) \in Q_{T} \text {. }
$$

The lower bound is easily obtained by using $n^{-}=\min (0, n)$ as test function in (4.8). To obtain the upper bound use $\left(n_{K}-M\right)^{+} \in L^{2}(V)$ as test function in (4.8). Since

$$
G(s) \stackrel{\text { def }}{=} \int_{0}^{s}\left(\tau_{K}-M\right)^{+} d \tau \geqslant \frac{1}{2}\left(\left(s_{K}-M\right)^{+}\right)^{2}
$$

we get for a.e. $t \in(0, T)[16$, p. 91$]$ 


$$
\begin{aligned}
\int_{0}^{t}\left\langle\partial_{t} n,\left(n_{K}-M\right)^{+}\right\rangle_{V^{*}, V}= & \int_{\Omega}(G(n(t))-G(n(0))) \\
& \geqslant \frac{1}{2} \int_{\Omega}\left(n_{K}-M\right)^{+}(t)^{2},
\end{aligned}
$$

and therefore,

$$
\begin{aligned}
& \frac{1}{2} \int_{\Omega}\left(n_{K}-M\right)^{+}(t)^{2}+\varepsilon \int_{Q_{t}}\left|\nabla\left(n_{K}-M\right)^{+}\right|^{2} \\
& \quad \leqslant-\int_{Q_{t}}\left(n_{K}-M\right) \nabla \phi \cdot \nabla\left(n_{K}-M\right)^{+}-M \int_{Q_{t}} \nabla \phi \cdot \nabla\left(n_{K}-M\right)^{+} \\
& \quad=-\frac{1}{2} \int_{Q_{t}} \nabla \phi \cdot \nabla\left(n_{K}-M\right)^{+2}-M \int_{Q_{t}} \nabla \phi \cdot \nabla\left(n_{K}-M\right)^{+} .
\end{aligned}
$$

Now use Eq. (4.9) for $\phi$ and the estimate (4.13):

$$
\begin{aligned}
& \frac{1}{2} \int_{\Omega}\left(n_{K}-M\right)^{+}(t)^{2} \\
& \quad \leqslant \frac{1}{2} \int_{Q_{t}}\left(f(\phi)-n_{M}\right)\left(n_{K}-M\right)^{+2}+M \int_{Q_{t}}\left(f(\phi)-n_{M}\right)\left(n_{K}-M\right)^{+} \\
& \quad \leqslant \frac{1}{2} \int_{Q_{t}}\left(M-n_{M}\right)\left(n_{K}-M\right)^{+2}+M \int_{Q_{t}}\left(M-n_{M}\right)\left(n_{K}-M\right)^{+} \\
& \quad=0,
\end{aligned}
$$

since $M-n_{M}=0$ in $\{n>M\}$ for all $K>M$. Thus, the estimate (4.15) is proved.

With these $L^{\infty}$ bounds for $n$ and $\phi$ independent of $K$ (and $\varepsilon$ ), we can remove the cut-off functions, and $\left(n^{\varepsilon}, \phi^{\varepsilon}\right)$ solves

$$
\begin{gathered}
\partial_{t} n^{\varepsilon}-\operatorname{div}\left(\nabla p^{\varepsilon}\left(n^{\varepsilon}\right)+n^{\varepsilon} \nabla \phi^{\varepsilon}\right)=0, \\
-\Delta \phi^{\varepsilon}=n^{\varepsilon}-f\left(\phi^{\varepsilon}\right) \quad \text { in } Q_{T}
\end{gathered}
$$

with the initial-boundary conditions (4.3)-(4.5), where $p^{\varepsilon}(s)=p(s)+$ $\varepsilon s$.

Third step: Further a priori estimates independent of $\varepsilon$. Taking $n^{\varepsilon}-n_{D}$ as test function in (4.16) gives 


$$
\begin{aligned}
& \frac{1}{2} \int_{\Omega}\left(n^{\varepsilon}-n_{D}\right)^{2}(t)+\int_{Q_{t}}\left(p^{\varepsilon}\right)^{\prime}\left(n^{\varepsilon}\right)\left|\nabla n^{\varepsilon}\right|^{2} \\
& =\int_{Q_{t}}\left(p^{\varepsilon}\right)^{\prime}\left(n^{\varepsilon}\right) \nabla n^{\varepsilon} \cdot \nabla n_{D}+\frac{1}{2} \int_{\Omega}\left(n^{\varepsilon}-n_{D}\right)^{2}(0) \\
& \quad-\int_{Q_{t}}\left(n^{\varepsilon}-n_{D}\right) \partial_{t} n_{D}-\int_{Q_{t}} n^{\varepsilon} \nabla \phi^{\varepsilon} \cdot \nabla\left(n^{\varepsilon}-n_{D}\right) .
\end{aligned}
$$

Since the last integral is equal to

$$
\begin{aligned}
& -\int_{Q_{t}}\left(n^{\varepsilon}-n_{D}\right) \nabla \phi^{\varepsilon} \cdot \nabla\left(n^{\varepsilon}-n_{D}\right)-\int_{Q_{t}} n_{D} \nabla \phi^{\varepsilon} \cdot \nabla\left(n^{\varepsilon}-n_{D}\right) \\
& =\frac{1}{2} \int_{Q_{t}} \Delta \phi^{\varepsilon}\left(n^{\varepsilon}-n_{D}\right)^{2}+\int_{Q_{t}} n_{D} \Delta \phi^{\varepsilon}\left(n^{\varepsilon}-n_{D}\right) \\
& \quad+\int_{Q_{t}} \nabla n_{D} \cdot \nabla \phi^{\varepsilon}\left(n^{\varepsilon}-n_{D}\right)
\end{aligned}
$$

and since the $L^{\infty}$-bounds for $n^{\varepsilon}$ and $\phi^{\varepsilon}$ imply, by using (4.17),

$$
\int_{Q_{t}}\left|\nabla \phi^{\varepsilon}\right|^{2} \leqslant c_{1}
$$

for some $c_{1}>0$ independent of $\varepsilon$, we get the $\varepsilon$-independent estimate

$$
-\int_{Q_{t}} n^{\varepsilon} \nabla \phi^{\varepsilon} \cdot \nabla\left(n^{\varepsilon}-n_{D}\right) \leqslant c .
$$

Here and in the following, $c$ denotes a positive constant independent of $\varepsilon$, with values varying from occurrence to occurrence. Therefore, we get from (4.18)

$$
\frac{1}{2} \int_{\Omega}\left(n^{\varepsilon}-n_{D}\right)(t)^{2}+\frac{1}{2} \int_{Q_{t}}\left(p^{\varepsilon}\right)^{\prime}\left(n^{\varepsilon}\right)\left|\nabla n^{\varepsilon}\right|^{2} \leqslant c+\int_{Q_{t}}\left(n^{\varepsilon}-n_{D}\right)^{2},
$$

and, employing Gronwall's lemma, we conclude

$$
\int_{\Omega}\left(n^{\varepsilon}-n_{D}\right)^{2}(t)+\int_{Q_{t}}\left(p^{\varepsilon}\right)^{\prime}\left(n^{\varepsilon}\right)\left|\nabla n^{\varepsilon}\right|^{2} \leqslant c(T),
$$


where $c(T)>0$ depends on $T$, but not on $\varepsilon$. The Lipschitz continuity of $p$ in $[0, M]$ implies

$$
\int_{Q_{T}}\left|\nabla p^{\varepsilon}\left(n^{\varepsilon}\right)\right|^{2} \leqslant c(M) \int_{Q_{T}}\left(p^{\varepsilon}\right)^{\prime}\left(n^{\varepsilon}\right)\left|\nabla n^{\varepsilon}\right|^{2} \leqslant c .
$$

Furthermore,

$$
\left\|\partial_{t} n^{\varepsilon}\right\|_{L^{2}\left(V^{*}\right)} \leqslant\left\|\nabla p^{\varepsilon}\left(n^{\varepsilon}\right)\right\|_{L^{2}\left(L^{2}\right)}+\left\|n^{\varepsilon}\right\|_{L^{\infty}\left(L^{\infty}\right)}\left\|\nabla \phi^{\varepsilon}\right\|_{L^{2}\left(L^{2}\right)} \leqslant c .
$$

Fourth step: The limit $\varepsilon \rightarrow 0$. From the estimates (4.19)-(4.21) and the second step follows the existence of a subsequence (not relabeled) such that

$$
\begin{aligned}
n^{\varepsilon} \stackrel{*}{\rightarrow} n & \text { weakly* in } L^{\infty}\left(Q_{T}\right), \\
\partial_{t} n^{\varepsilon} \rightarrow \partial_{t} n & \text { weakly in } L^{2}\left(V^{*}\right), \\
\nabla p^{\varepsilon}\left(n^{\varepsilon}\right) \rightarrow \nabla \rho & \text { weakly in } L^{2}\left(Q_{T}\right), \\
\nabla \phi^{\varepsilon} \rightarrow \nabla \phi & \text { weakly in } L^{2}\left(Q_{T}\right) \text { as } \varepsilon \rightarrow 0,
\end{aligned}
$$

for some functions $n \in L^{\infty}\left(Q_{T}\right) \cap H^{1}\left(V^{*}\right), \quad \rho \in L^{2}\left(H^{1}\right)$, and $\phi \in$ $L^{2}\left(H^{1}\right)$. The identification $\rho=p(n)$ follows from the usual monotonicity argument. Indeed, setting $p_{M}(s)=\min (p(M), \max (0, p(s)))$, the operator $P: L^{2}\left(Q_{T}\right) \rightarrow\left(L^{2}\left(Q_{T}\right)\right)^{*},\langle P(u), v\rangle=\int_{Q_{T}} p_{M}(u) v$, is monotone (thanks to $\left(\mathrm{A} 2^{\prime}\right)$ ) and hemicontinuous (since $p_{M}$ is continuous and bounded), hence maximal monotone. Since $\left(n^{\varepsilon}\right)$ is bounded in $L^{\infty}\left(L^{2}\right)$ and in $H^{1}\left(V^{*}\right)$ and the embedding $L^{2}(\Omega) \hookrightarrow V^{*}$ is compact, we infer from Aubin's lemma [26] that

$$
n^{\varepsilon} \rightarrow n \quad \text { in } C^{0}\left([0, T] ; V^{*}\right)
$$

The strong convergence of $n^{\varepsilon}$ to $n$ in $L^{2}\left(V^{*}\right)$ and the weak convergence of $p^{\varepsilon}\left(n^{\varepsilon}\right)$ to $\rho$ in $L^{2}(V)$ together with the monotonicity of $p^{\varepsilon}$ applied to the inequality

$$
\int_{0}^{T}\left\langle n^{\varepsilon}-v, p^{\varepsilon}\left(n^{\varepsilon}\right)-p^{\varepsilon}(v)\right\rangle_{V^{*}, V} \geqslant 0 \quad \text { for all } v \in C_{0}^{\infty}\left(Q_{T}\right)+n_{D}
$$


give

$$
\langle n-v, \rho-P(v)\rangle_{L^{2}, L^{2}} \geqslant 0 \quad \text { for all } v \in L^{2}\left(Q_{T}\right)+n_{D} .
$$

Since $P$ is maximal monotone, we conclude $\rho=p(n)$.

Next we show that

$$
\nabla \phi^{\varepsilon} \rightarrow \nabla \phi \quad \text { in } L^{2}\left(Q_{T}\right) .
$$

We proceed similarly as in the proof of Theorem 2.2. We use $\phi^{\varepsilon}-\phi$ as test function in Eq. (4.17) and use the monotonicity of $f$ to obtain

$$
\begin{aligned}
\int_{Q_{T}}\left|\nabla \phi^{\varepsilon}\right|^{2}= & \int_{Q_{T}} \nabla \phi^{\varepsilon} \cdot \nabla \phi+\int_{Q_{T}}\left(n^{\varepsilon}-f(\phi)\right)\left(\phi^{\varepsilon}-\phi\right) \\
& -\int_{Q_{T}}\left(f\left(\phi^{\varepsilon}\right)-f(\phi)\right)\left(\phi^{\varepsilon}-\phi\right) \\
\leqslant & \int_{Q_{T}} \nabla \phi^{\varepsilon} \cdot \nabla \phi+\int_{Q_{T}}\left(n^{\varepsilon}-n\right)\left(\phi^{\varepsilon}-\phi\right) \\
& +\int_{Q_{T}}(n-f(\phi))\left(\phi^{\varepsilon}-\phi\right) .
\end{aligned}
$$

The strong convergence of $n^{\varepsilon}$ in $L^{2}\left(V^{*}\right)$ and the weak convergence of $\phi^{\varepsilon}$ in $L^{2}(V)$ give

$$
\limsup _{\varepsilon \rightarrow 0} \int_{Q_{T}}\left|\nabla \phi^{\varepsilon}\right|^{2} \leqslant \int_{Q_{T}}|\nabla \phi|^{2} .
$$

Therefore, together with the inequality

$$
\int_{Q_{T}}|\nabla \phi|^{2} \leqslant \liminf _{\varepsilon \rightarrow 0} \int_{Q_{T}}\left|\nabla \phi^{\varepsilon}\right|^{2},
$$

which follows from the weak convergence of $\nabla \phi^{\varepsilon}$ in $L^{2}\left(Q_{T}\right)$, we get

$$
\lim _{\varepsilon \rightarrow 0} \int_{Q_{T}}\left|\nabla \phi^{\varepsilon}\right|^{2}=\int_{Q_{T}}|\nabla \phi|^{2} .
$$

The convergence of the norms and the weak convergence imply the strong convergence, i.e., (4.22). 
Now we can pass to the limit $\varepsilon \rightarrow 0$ in the weak formulation of (4.16), using the weak convergences of $n^{\varepsilon}$ to $n$ and $p^{\varepsilon}\left(n^{\varepsilon}\right)$ to $p(n)$ in $L^{2}\left(Q_{T}\right)$ and the strong convergence (4.22), to conclude that $(n, \phi)$ solves (4.1)(4.2). Furthermore, $(n, \phi)$ satisfies the boundary conditions (4.3)-(4.4) in the sense of (4.6), and $n(0)=n_{I}$ since $n \in C^{0}\left([0, T] ; V^{*}\right)$.

We end this section by proving some positivity results on $n$ which are needed for the uniqueness result. We call $(n, \phi)$ a limit solution of (4.1)(4.5) if it is obtained as the ( $L^{2}$-weak) limit of approximate solutions $\left(n^{\varepsilon}, \phi^{\varepsilon}\right) \in\left(L^{\infty}\left(Q_{T}\right) \cap L^{2}\left(H^{1}\right) \cap H^{1}\left(V^{*}\right)\right)^{2}$ of the problem (4.16)-(4.17) subject to the initial-boundary conditions (4.3)-(4.5).

We show now that the ion density (of every limit solution) remains positive (at least for finite time), if the initial and boundary densities are positive, even in the adiabatic case. Let us suppose in the following that the assumptions (A1), (A2')-(A4') hold.

PROPOSITION 4.2. - Suppose that there exist constants $n_{0}>0, \lambda_{0} \geqslant$ 0 such that

$$
n_{I}(x) \geqslant n_{0} \quad \text { in } \Omega, \quad n_{D}(x, t) \geqslant n_{0} \mathrm{e}^{-\lambda_{0} t} \quad \text { on } \Gamma_{D} \times(0, T),
$$

where $T=\infty$ is admissible. Then there exists a constant $\lambda_{1} \geqslant \lambda_{0}>0$ such that for every limit solution it holds

$$
n(x, t) \geqslant n_{0} \mathrm{e}^{-\lambda_{1} t} \quad \text { in } \Omega \times(0, T) .
$$

Proof. - Let $\left(n^{\varepsilon}, \phi^{\varepsilon}\right)$ be a solution to the approximate problem (4.16)(4.17), (4.3)-(4.5) such that $n^{\varepsilon} \rightarrow n$ weakly in $L^{2}\left(Q_{T}\right)$. In the proof of Theorem 4.1 we have shown that (see (4.7))

$$
0 \leqslant n^{\varepsilon}(x, t) \leqslant M_{T}, \quad \phi^{\varepsilon}(x, t) \geqslant \underline{\phi} \quad \text { in } Q_{T} .
$$

Let $z=n_{0} \exp \left(-\lambda_{1} t\right)$, where $\lambda_{1} \geqslant \lambda_{0}$ will be specified later. Using $\left(n^{\varepsilon}-z\right)^{-} \in L^{2}(V)$ as test function in (4.16) and employing (4.17), we get

$$
\begin{aligned}
& \frac{1}{2} \int_{\Omega}\left(n^{\varepsilon}-z\right)^{-}(t)^{2}+\int_{Q_{t}}\left(p^{\varepsilon}\right)^{\prime}\left(n^{\varepsilon}\right)\left|\nabla\left(n^{\varepsilon}-z\right)^{-}\right|^{2} \\
& =\lambda_{1} \int_{Q_{t}} z\left(n^{\varepsilon}-z\right)^{-}-\int_{Q_{t}}\left(n^{\varepsilon}-z\right) \nabla \phi^{\varepsilon} \cdot \nabla\left(n^{\varepsilon}-z\right)^{-}
\end{aligned}
$$




$$
\begin{aligned}
& -\int_{Q_{t}} z \nabla \phi^{\varepsilon} \cdot \nabla\left(n^{\varepsilon}-z\right)^{-} \\
= & \lambda_{1} \int_{Q_{t}} z\left(n^{\varepsilon}-z\right)^{-}+\frac{1}{2} \int_{Q_{t}} \Delta \phi^{\varepsilon}\left(n^{\varepsilon}-z\right)^{-2}+\int_{Q_{t}} z \Delta \phi^{\varepsilon}\left(n^{\varepsilon}-z\right)^{-} \\
\leqslant & \frac{1}{2} \int_{Q_{t}} f\left(\phi^{\varepsilon}\right)\left(n^{\varepsilon}-z\right)^{-2}+\int_{Q_{t}}\left(\lambda_{1}+f\left(\phi^{\varepsilon}\right)-n^{\varepsilon}\right) z\left(n^{\varepsilon}-z\right)^{-} \\
\leqslant & \frac{M_{T}}{2} \int_{Q_{t}}\left(n^{\varepsilon}-z\right)^{-2}+\int_{Q_{t}}\left(\lambda_{1}+f(\phi)-M_{T}\right) z\left(n^{\varepsilon}-z\right)^{-} \\
\leqslant & \frac{M_{T}}{2} \int_{Q_{t}}\left(n^{\varepsilon}-z\right)^{-2},
\end{aligned}
$$

if we take $\lambda_{1} \geqslant \max \left(\lambda_{0}, M_{T}-f(\phi)\right)$. Now, Gronwall's lemma implies $\left(n^{\varepsilon}-z\right)^{-}(t)=0$ in $\Omega$, for a.e. $\left.t \in \overline{(0}, T\right)$. Therefore, $n^{\varepsilon}(x, t) \geqslant z$ in $Q_{T}$, and the conclusion follows after letting $\varepsilon \rightarrow 0$.

The above proposition can be improved if the function $f$ is uniformly positive, including the case $f=\exp$.

Proposition 4.3. - Suppose that $f(s)>0$ for all $s \in \mathbb{R}$ and that

$$
n_{I} \geqslant n_{0} \quad \text { in } \Omega, \quad n_{D} \geqslant n_{0} \quad \text { on } \Gamma_{D} \times(0, \infty),
$$

for some $n_{0}>0$. Then there exists a constant $\underline{n}>0$ such that, for every limit solution,

$$
n(x, t) \geqslant \underline{n} \text { in } \Omega \times(0, \infty) .
$$

Proof. - Let $\left(n^{\varepsilon}, \phi^{\varepsilon}\right)$ be as in the proof of Proposition 4.2. From (4.23) follows that $f\left(\phi^{\varepsilon}\right) \geqslant f(\phi)>0$ in $Q_{T}$. Set $\underline{n}=\min \left(n_{0}, f(\phi)\right)>0$. Using $\left(n^{\varepsilon}-\underline{n}\right)^{-}$as test function in (4.16) and proceeding as in the proof of Proposition 4.2, we obtain

$$
\begin{aligned}
\frac{1}{2} \int_{\Omega}\left(n^{\varepsilon}-\underline{n}\right)^{-}(t)^{2} & \leqslant \frac{1}{2} \int_{Q_{T}} \Delta \phi^{\varepsilon}\left(n^{\varepsilon}-\underline{n}\right)^{-2}+\underline{n} \int_{Q_{T}} \Delta \phi^{\varepsilon}\left(n^{\varepsilon}-\underline{n}\right)^{-} \\
& =\frac{1}{2} \int_{Q_{T}}\left(f\left(\phi^{\varepsilon}\right)-n^{\varepsilon}\right)\left(n^{\varepsilon}+\underline{n}\right)\left(n^{\varepsilon}-\underline{n}\right)^{-} \\
& \leqslant \frac{1}{2} \int_{Q_{T}}(f(\underline{\phi})-\underline{n})\left(n^{\varepsilon}+\underline{n}\right)\left(n^{\varepsilon}-\underline{n}\right)^{-} \leqslant 0 .
\end{aligned}
$$


Therefore, $n^{\varepsilon} \geqslant \underline{n}$ in $Q_{T}$, from which we conclude the assertion.

\section{UNIQUENESS OF SOLUTIONS}

We present two results on the uniqueness of solutions to (4.1)-(4.5). For both results we need additional assumptions. First, we prove the uniqueness of solutions in the class of weak solutions (as defined in Section 4) if isothermal states are assumed, i.e., $p(n)=n$.

For nonlinear pressure functions, the problem becomes quasilinear, which is more delicate. We show the uniqueness of solutions in the class of weak solutions satisfying $\phi \in L^{\infty}\left(W^{1, \infty}\right)$, where we have to exclude mixed boundary conditions. Indeed, it is well known that in the case of mixed Dirichlet-Neumann boundary conditions, we get, in general, at most $\phi(t) \in W^{2,4 / 3-\varepsilon}(\Omega)$ for every $\varepsilon>0$ [2], even for smooth data. For the second result, we also have to assume that either $p^{\prime}(s)$ is strictly positive or the initial and boundary densities are strictly positive. This condition implies that the problem (4.1)-(4.5) is uniformly parabolic.

We cannot present a general uniqueness result for degenerate problems. The reason lies in the fact that we are not able to deal with both the degeneracy of the function $p$ and with the nonlocal drift term. It is possible to prove uniqueness of solutions of the degenerate problem for given $\phi$ (see, e.g., [13] for a related result) or of the nondegenerate problem coupled self-consistently to the Poisson equation (Theorem 5.2). In semiconductor modeling and in the hydrodynamics of immiscible fluids through a porous medium, similar difficulties occur and no general uniqueness results are available (see [1,7,8]). We refer to [7] for related results for the degenerate case under special conditions (also see Remark 5.3).

We assume throughout this section that the conditions (A1), (A2')(A4') hold.

THEOREM 5.1. - Let $p(s)=s$ for $s \geqslant 0$. Then there exists a unique weak solution $(n, \phi)$ of (4.1)-(4.5).

Proof. - Let $\left(n_{1}, \phi_{1}\right)$ and $\left(n_{2}, \phi_{2}\right)$ be two solutions to (4.1)-(4.5). Then $n_{1}, n_{2} \in L^{2}\left(H^{1}\right) \cap H^{1}\left(V^{*}\right) \hookrightarrow C^{0}\left([0, T] ; L^{2}(\Omega)\right)$. Using $n_{1}-n_{2}$ as test function in the difference of Eqs. (4.1), satisfied by $\left(n_{1}, \phi_{1}\right),\left(n_{2}, \phi_{2}\right)$, respectively, we obtain 


$$
\begin{aligned}
& \frac{1}{2} \int_{\Omega}\left(n_{1}-n_{2}\right)(t)^{2}+\int_{Q_{t}}\left|\nabla\left(n_{1}-n_{2}\right)\right|^{2} \\
& =\int_{Q_{t}}\left(n_{1}-n_{2}\right) \nabla \phi_{1} \cdot \nabla\left(n_{1}-n_{2}\right)+\int_{Q_{t}} n_{2} \nabla\left(\phi_{1}-\phi_{2}\right) \cdot \nabla\left(n_{1}-n_{2}\right) \\
& \leqslant \frac{1}{2} \int_{Q_{t}}\left(n_{1}-f\left(\phi_{1}\right)\right)\left(n_{1}-n_{2}\right)^{2}+\frac{1}{2} \int_{Q_{t}}\left|\nabla\left(n_{1}-n_{2}\right)\right|^{2} \\
& \quad+\frac{1}{2} \int_{Q_{t}} n_{2}^{2}\left|\nabla\left(\phi_{1}-\phi_{2}\right)\right|^{2}
\end{aligned}
$$

for every $t \in(0, T)$. Here, we have used Eq. (4.2) and Young's inequality. Observing that, thanks to the monotonicity of $f$,

$$
\int_{Q_{t}}\left|\nabla\left(\phi_{1}-\phi_{2}\right)\right|^{2} \leqslant \int_{Q_{t}}\left(n_{1}-n_{2}\right)^{2},
$$

and taking into account the $L^{\infty}$ bounds (4.7), we get

$$
\begin{aligned}
& \frac{1}{2} \int_{\Omega}\left(n_{1}-n_{2}\right)(t)^{2}+\frac{1}{2} \int_{Q_{t}}\left|\nabla\left(n_{1}-n_{2}\right)\right|^{2} \\
& \quad \leqslant \frac{1}{2}\left[\left(M_{T}-f(\underline{\phi})\right)+M_{T}^{2}\right] \int_{Q_{t}}\left(n_{1}-n_{2}\right)^{2} .
\end{aligned}
$$

We conclude from Gronwall's lemma that $\left(n_{1}-n_{2}\right)(t)=0$ a.e. in $\Omega$, for every $t \in(0, T)$.

THEOREM 5.2. - Let $\partial \Omega=\Gamma_{D} \in C^{2+\varepsilon}(\varepsilon>0), \phi_{D} \in L^{\infty}\left(W^{2, q}\right)$ for $q>d$ ( $d$ being the space dimension), $p$ is strictly increasing, $f$ is locally Lipschitz continuous in $\mathbb{R}$, and either

(i) $p^{\prime}(s) \geqslant p_{0}>0$ for all $s \geqslant 0$, or

(ii) $n_{D} \geqslant n_{0}>0$ on $\Gamma_{D} \times(0, T), n_{I} \geqslant n_{0}>0$ in $\Omega$.

Then there exists a unique weak solution of (4.1)-(4.5) satisfying $\phi \in$ $L^{\infty}\left(W^{2, q}\right)$.

Proof. - Let $\left(n_{1}, \phi_{1}\right)$ be a limit solution to (4.1)-(4.5) (see the definition before Proposition 4.2) and let $\left(n_{2}, \phi_{2}\right)$ be another weak solution. Using elliptic regularity theory [28], we conclude that $\phi_{i}(t) \in W^{2, q}(\Omega)$ and $\nabla \phi_{i} \in L^{\infty}\left(Q_{T}\right)$ for $i=1,2$, since $q>d$. Set $n=n_{1}-n_{2}, \phi=\phi_{1}-\phi_{2}$, and let $\tau>0$. We get for every test function $\psi \in C^{\infty}\left(\bar{Q}_{T}\right)$ satisfying $\left.\psi\right|_{\partial \Omega}=0$ and $\psi(\tau)=0$ in $\Omega$ 


$$
\begin{gathered}
-\int_{Q_{\tau}} n \partial_{t} \psi-\int_{Q_{\tau}}\left(p\left(n_{1}\right)-p\left(n_{2}\right)\right) \Delta \psi \\
=-\int_{Q_{\tau}}\left(n \nabla \phi_{1} \cdot \nabla \psi+n_{2} \nabla \phi \cdot \nabla \psi\right) .
\end{gathered}
$$

Now set

$$
A(x, t)=\int_{0}^{1} p^{\prime}\left(\theta n_{1}+(1-\theta) n_{2}\right) \mathrm{d} \theta .
$$

If the condition (5.2) is satisfied then it holds $A \geqslant p_{0}>0$ in $Q_{T}$. In the case of condition (5.2) we get the existence of $\underline{n}>0$ such that $n_{1}(x, t) \geqslant \underline{n}$ in $Q_{T}$, by Proposition 4.2, and therefore,

$$
A \geqslant \int_{0}^{1} p^{\prime}(\theta \underline{n}) \mathrm{d} \theta=(p(\underline{n})-p(0)) / \underline{n}>0 \quad \text { in } Q_{T} .
$$

Furthermore, we get the regularity $n_{1}, n_{2} \in C^{0}\left([0, T] ; L^{2}(\Omega)\right)$.

There exist sequences $A_{\eta}, B_{\eta} \in C_{0}^{\infty}\left(Q_{T}\right)$ such that

$$
\begin{aligned}
& A_{\eta} \rightarrow A \quad \text { in } L^{2}\left(Q_{T}\right), \quad \sup \left\{\left\|A_{\eta}\right\|_{0, \infty}: \eta>0\right\}<\infty, \\
& \inf \left\{A_{\eta}: \eta>0\right\}>0, \\
& B_{\eta} \rightarrow \nabla \phi_{1} \quad \text { in } L^{2}\left(Q_{T}\right) \text { as } \eta \rightarrow 0, \\
& \sup \left\{\left\|B_{\eta}\right\|_{0, \infty}: \eta>0\right\}<\infty .
\end{aligned}
$$

We rewrite Eq. (5.1):

$$
\begin{aligned}
& -\int_{Q_{\tau}} n\left(\partial_{t} \psi+A_{\eta} \Delta \psi-B_{\eta} \cdot \nabla \psi\right) \\
& \quad=\int_{Q_{\tau}} n\left(A-A_{\eta}\right) \Delta \psi-\int_{Q_{\tau}} n\left(\nabla \phi_{1}-B_{\eta}\right) \cdot \nabla \psi-\int_{Q_{\tau}} n_{2} \nabla \phi \cdot \nabla \psi .
\end{aligned}
$$

Let $\psi=\psi_{\eta} \in C^{\infty}\left(\bar{Q}_{T}\right)$ be the unique solution of the retrograde uniformly parabolic problem

$$
\begin{gathered}
\partial_{t} \psi+A_{\eta} \Delta \psi-B_{\eta} \cdot \nabla \psi=-g_{\eta} \quad \text { in } Q_{\tau}, \\
\psi=0 \quad \text { on } \partial \Omega \times(0, \tau), \quad \psi(\tau)=0 \quad \text { in } \Omega,
\end{gathered}
$$

where $g_{\eta} \in C_{0}^{\infty}\left(Q_{\tau}\right)$ satisfies $g_{\eta} \rightarrow n$ in $L^{2}\left(Q_{\tau}\right)$. The existence of $\psi_{\eta}$ follows from standard parabolic theory [18]. Multiplying the differential 
equation (5.3) by $\Delta \psi_{\eta}$, we easily get the following estimate (see, e.g., [13] for details):

$$
\left\|\nabla \psi_{\eta}\right\|_{L^{\infty}\left(L^{2}\right)}+\left\|\Delta \psi_{\eta}\right\|_{L^{2}\left(L^{2}\right)} \leqslant c_{0}\left\|g_{\eta}\right\|_{L^{2}\left(L^{2}\right)},
$$

where $c_{0}>0$ depends on $p_{0}$ in case (i) or on $\underline{u}$ in case (ii). Observing that

$$
\left\|\nabla \psi_{\eta}\right\|_{0,2, Q_{\tau}} \leqslant \sqrt{\tau}\left\|\nabla \psi_{\eta}\right\|_{L^{\infty}\left(L^{2}\right)},
$$

we get from Eq. (5.2)

$$
\begin{aligned}
\int_{Q_{\tau}} n g_{\eta} \leqslant & \|n\|_{0, \infty}\left\|A-A_{\eta}\right\|_{0,2}\left\|\Delta \psi_{\eta}\right\|_{0,2}+\|n\|_{0, \infty} \| \nabla \phi_{1} \\
& -B_{\eta}\left\|_{0,2}\right\| \nabla \psi_{\eta}\left\|_{0,2}+c_{0} \sqrt{\tau}\right\| n_{2}\left\|_{0, \infty}\right\| \nabla \phi\left\|_{0,2}\right\| g_{\eta} \|_{0,2} .
\end{aligned}
$$

Performing the limit $\eta \rightarrow 0$ yields

$$
\int_{Q_{\tau}} n^{2} \leqslant c_{0} M \sqrt{\tau}\|\nabla \phi\|_{0,2, Q_{\tau}}\|n\|_{0,2, Q_{\tau}},
$$

where $M=\left\|n_{2}\right\|_{0, \infty, Q_{\tau}}$. The difference $\phi=\phi_{1}-\phi_{2}$ satisfies, since $f$ is monotone, the equation

$$
\int_{Q_{\tau}}|\nabla \phi|^{2} \leqslant \int_{Q_{\tau}} n \phi \leqslant c_{1}\|n\|_{0,2, Q_{\tau}}\|\nabla \phi\|_{0,2, Q_{\tau}},
$$

which implies, together with (5.4),

$$
\|n\|_{0,2, Q_{\tau}}^{2}=\int_{Q_{\tau}} n^{2} \leqslant c_{0} c_{1} M \sqrt{\tau}\|n\|_{0,2, Q_{\tau}}^{2} .
$$

Choosing $\tau<1 /\left(c_{0} c_{1} M\right)^{2}$, we conclude that $n_{1}-n_{2}=0$ a.e. in $Q_{\tau}$. In particular, $\left(n_{1}-n_{2}\right)(\tau)=0$ in the sense of $V^{*}$. Thanks to (5.5) we infer that $\phi_{1}-\phi_{2}=0$ a.e. in $Q_{\tau}$. Applying the above method to the problem (4.1)-(4.5) in $\Omega \times(\tau, 2 \tau)$, we get $n_{1}-n_{2}=0$ and $\phi_{1}-\phi_{2}=0$ a.e. in $\Omega \times(\tau, 2 \tau)$ with $\left(u_{1}-u_{2}\right)(2 \tau)=0$ in the sense of $V^{*}$, etc. Finally, after a finite number of steps we arrive to $n_{1}=n_{2}$ and $\phi_{1}=\phi_{2}$ a.e. in $Q_{T}$.

Remark 5.3. - It is possible to prove the uniqueness of solutions of the mixed boundary-value problem in one or two space dimensions, provided 
that the boundary $\partial \Omega$ is Lipschitzian and that $n_{I} \in W^{1, p}(\Omega)$ and $n_{D}$, $\phi_{D} \in L^{\infty}\left(W^{1, p}\right)$ for some $p>2$. This result can be proved as in [11].

Furthermore, it is possible to relax the hypothesis that $p(s)$ is strictly increasing. Indeed, we get uniqueness of solutions, provided that $p(s)$ is only non-decreasing and that the inequality

$$
\nabla \phi \cdot v \leqslant 0 \quad \text { on } \Gamma_{D} \times(0, T)
$$

is satisfied for some solution $(n, \phi)$. This inequality can be interpreted as an entropy-type condition, since Eq. (4.1) becomes hyperbolic if $p(s)=$ const. The proof of this result is analogous to the corresponding proof in [6].

\section{ACKNOWLEDGEMENTS}

The first author acknowledges partial support from the DAADPROCOPE Program, from the Deutsche Forschungsgemeinschaft, grant numbers MA 1662/-1 and -2, and from the TMR Project "Asymptotic Methods in Kinetic Theory”, grant number ERB-FMBX-CT97-0157. The research of the second author was partially supported by the TMR network of the project "Hyperbolic Systems of Conservation Laws" (HCL), grant number ERB-FMRX-CT96-0033. The first author would like to thank Naoufel Ben Abdallah (Toulouse, France) for valuable discussions.

\section{APPENDIX A}

The hydrodynamic plasma equations including the physical parameters read as follows:

$$
\begin{aligned}
& \partial_{t} n_{\alpha}+\operatorname{div}\left(n_{\alpha} u_{\alpha}\right)=0, \quad \alpha=e, i, \\
& \partial_{t}\left(m_{\alpha} n_{\alpha} u_{\alpha}\right)+\operatorname{div}\left(m_{\alpha} n_{\alpha} u_{\alpha} \otimes u_{\alpha}\right)+\nabla p_{\alpha}\left(n_{\alpha}\right) \\
& \quad=Q_{\alpha} n_{\alpha} \nabla \phi-\frac{m_{\alpha} n_{\alpha} u_{\alpha}}{\tau_{\alpha}}, \\
& -\varepsilon_{0} \Delta \phi=q\left(n_{i}-n_{e}\right),
\end{aligned}
$$

where $Q_{e}=-q, Q_{i}=+q, q$ is the elementary charge, and $\varepsilon_{0}$ is the permittivity constant. For the scaling, we assume that the electron mass $m_{e}$, the ion mass $m_{i}$ and the relaxation times $\tau_{e}, \tau_{i}$ are constant. Let $L$ denote the diameter of the domain, let $N_{\alpha}$ and $v_{\alpha}$ be typical density 
and velocity values for the electrons and ions, respectively $(\alpha=e, i)$.

Furthermore, we set

$$
\tau_{0}=L / v_{0}, \quad \tau_{0, \alpha}=L / v_{\alpha}, \quad \alpha=e, i,
$$

where $v_{0}$ is a velocity constant, and we define the scaled Debye length

$$
\lambda=\sqrt{\frac{\varepsilon_{0} k_{B} T_{0}}{q^{2} L^{2} \max \left(N_{e}, N_{i}\right)}},
$$

where $T_{0}$ is a temperature constant. Then, performing the scaling

$$
\begin{aligned}
x \rightarrow L x, \quad & t \rightarrow \tau_{0} t, \quad \tau_{\alpha} \rightarrow \tau_{0, \alpha} \tau_{\alpha}, \\
n_{\alpha} \rightarrow N_{\alpha} n_{\alpha}, \quad u_{\alpha} \rightarrow & v_{\alpha} u_{\alpha}, \quad p_{\alpha}\left(n_{\alpha}\right) \rightarrow k_{B} T_{0} N_{\alpha} p_{\alpha}\left(n_{\alpha}\right), \\
\phi & \rightarrow\left(k_{B} T_{0} / q\right) \phi,
\end{aligned}
$$

in the system (A.1)-(A.3), we get the equations for the dimensionless variables and parameters

$$
\begin{aligned}
& \partial_{t} n_{\alpha}+\left(v_{\alpha} / v_{0}\right) \operatorname{div}\left(n_{\alpha} u_{\alpha}\right)=0, \\
& \delta_{\alpha}\left(v_{0} / v_{\alpha}\right) \partial_{t}\left(n_{\alpha} u_{\alpha}\right)+\delta_{\alpha} \operatorname{div}\left(n_{\alpha} u_{\alpha} \otimes u_{\alpha}\right)+\nabla p_{\alpha}\left(n_{\alpha}\right) \\
& \quad=-q_{\alpha} n_{\alpha} \nabla \phi-\delta_{\alpha} \frac{n_{\alpha} u_{\alpha}}{\tau_{\alpha}}, \\
& -\lambda^{2} \Delta \phi=\gamma_{i} n_{i}-\gamma_{e} n_{e} .
\end{aligned}
$$

Here, $q_{e}=-1, q_{i}=+1, \gamma_{\alpha}=N_{\alpha} / \max \left(N_{e}, N_{i}\right)$, and

$$
\delta_{\alpha}=\frac{m_{\alpha} v_{\alpha}^{2}}{k_{B} T_{0}}, \quad \alpha=e, i .
$$

In order to obtain the model (HD-EI) of Section 1, set $v_{0}=v_{e}=v_{i}$ and $N_{e}=N_{i}$ such that $\gamma_{e}=\gamma_{i}=1$.

\section{REFERENCES}

[1] S. Antontsev, A. Domansky and J.I. Díaz, Continuous dependence and stabilization of solutions of the degenerate system in two-phase filtration, Dinamika Sploshnoi Sredy 107 (1993) 11-25.

[2] H. BEIRAO DA VEIGA, On the $W^{2, p}$-regularity for solutions of mixed problems, J. Math. Pures Appl. 53 (1974) 279-290.

[3] H. BRÉZIS, Convergence in $\mathcal{D}^{\prime}$ and in $L^{1}$ under strict convexity, in: J.-L. Lions (Ed.), Boundary Value Problems for Partial Differential Equations and Applications, Res. Notes Appl. Math. 29, Masson, 1993, pp. 43-52. 
[4] S. CORDIER, Global solutions to the isothermal Euler-Poisson plasma model, Appl. Math. Lett. 8 (1994) 19-24.

[5] S. Cordier and Y.-J. Peng, Système Euler-Poisson non linéaire-existence globale de solutions faibles entropiques, Mod. Math. Anal. Num. 32 (1998) 1-23.

[6] J.I. DíAZ, G. GALIANO and A. JÜNGEL, On a quasilinear degenerate system arising in semiconductor theory, Part I: Existence and uniqueness of solutions, To appear in Nonlin. Anal. TMA (2000).

[7] J.I. DíaZ, G. Galiano and A. JÜNGEL, Space localization and uniqueness of vacuum solutions to a degenerate parabolic problem in semiconductor theory, C. R. Acad. Sci. Paris 325 (1997) 267-272.

[8] G. Gagneux and M. Madaune-Tort, Sur la question de l'unicité pour les inéquations des milieux poreux, C. R. Acad. Sci. Paris 314 (1992) 605-608.

[9] C. GARDNER, Numerical simulation of a steady-state electron shock wave in a submicron semiconductor device, IEEE Trans. El. Dev. 38 (1991) 392-398.

[10] T. Goudon, A. JÜngel and Y.-J. PENG, Zero-electron-mass limits in hydrodynamic models for plasmas, Appl. Math. Lett. 12 (1999) 75-79.

[11] K. GRÖGER and J. REHBERG, Uniqueness for the two-dimensional semiconductor equations in case of high carrier densities, Math. Z. 213 (1993) 523-530.

[12] X. JIANG, A streamline-upwinding/Petrov-Galerkin method for the hydrodynamic semiconductor device model, Math. Models Meth. Appl. Sci. 5 (1995) 659-681.

[13] A. JÜNGEL, On the existence and uniqueness of transient solutions of a degenerate nonlinear drift-diffusion model for semiconductors, Math. Models Meth. Appl. Sci. 4 (1994) 677-703.

[14] A. JÜNGEL, Numerical approximation of a drift-diffusion model for semiconductors with nonlinear diffusion, Z. Angew. Math. Mech. 75 (1995) 783-799.

[15] A. JÜNGEL, Qualitative behavior of solutions of a degenerate nonlinear driftdiffusion model for semiconductors, Math. Models Meth. Appl. Sci. 5 (1995) 497518.

[16] A. JÜNGEL, A nonlinear drift-diffusion system with electric convection arising in semiconductor and electrophoretic modeling, Math. Nachr. 185 (1997) 85-110.

[17] A. JÜNGEL and Y.-J. PENG, A hierarchy of hydrodynamic plasma models. Zerorelaxation-time limits, Comm. P. D. E. 24 (1999) 1007-1033.

[18] O.A. LAdYZEnSKaya, V.A. Solonnikov and N.N. URAL'CEVA, Linear and Quasilinear Equations of Parabolic Type, Amer. Math. Soc., Providence, RI, 1968.

[19] P.A. Markowich, The Stationary Semiconductor Device Equations, Springer, Wien, 1986.

[20] P. MarCati and R. NATAlini, Weak solutions to a hydrodynamic model for semiconductors: The Cauchy problem, Proc. Roy. Soc. Edinburgh Sect. A 125 (1995) 115-131.

[21] P. Marcati and R. NATAlini, Weak solutions to a hydrodynamic model for semiconductors and relaxation to the drift-diffusion equation, Arch. Rat. Mech. Anal. 129 (1995) 129-145.

[22] R. NATALINI, The bipolar hydrodynamic model for semiconductors and the driftdiffusion equation, J. Math. Anal. Appl. 198 (1996) 262-281.

[23] Y.-J. PENG, Convergence of the fractional step Lax-Friedrichs scheme and Godunov scheme for a nonlinear Euler-Poisson system, Nonlin. Anal. (1999) (to appear). 
[24] F. Poupaud, M. RASCLE and J. Vila, Global solutions to the isothermal EulerPoisson system with arbitrarily large data, J. Differential Equations 123 (1995) 93-121.

[25] P. RAVIART, On singular perturbation problems for the nonlinear Poisson equation or: A mathematical approach to electrostatic sheaths and plasma erosion, Lecture Notes of the Summer School in Ile d'Oléron, France, 1997, pp. 452-539.

[26] J. Simon, Compact sets in the space $L^{p}(0, T ; B)$, Ann. Math. Pura Appl. 146 (1987) 65-96.

[27] G. Stampacchia, Equations Elliptiques du Second Ordre à Coefficients Discontinus, Les Presses de l’Université de Montréal, Canada, 1966.

[28] G.M. Troianiello, Elliptic Differential Equations and Obstacle Problems, Plenum Press, New York, 1987.

[29] A. Visintin, Strong convergence results related to strict convexity, Comm. Partial Differential Equations 9 (1984) 439-466.

[30] E. ZEIDLER, Nonlinear Functional Analysis and its Applications, Vol. II, Springer, New York, 1990. 